\title{
MULTIFRACTAL SPECTRA OF MORAN MEASURES WITHOUT LOCAL DIMENSION
}

\author{
ZHIHUI YUAN
}

\begin{abstract}
A measure without local dimension is a measure such that local dimension does not exist for any point in its support. In this paper, we construct such a class of Moran measures and study their lower and upper local dimensions. We show that the related "free energy" function ( $L^{q}$-spectrum) does not exist. Nevertheless, we can obtain the full Hausdroff and packing dimension spectra for level sets defined by lower and upper local dimensions. They can be viewed as a generalized multifractal formalism.
\end{abstract}

\section{INTRODUCTION}

Multifractal analysis is a natural framework to finely describe geometrically the heterogeneity in the distribution at small scales of the measures on a metric space. The multifractal formalism aims at expressing the dimension of the level sets in terms of the Legendre transform of some "free energy" function. The general setting is as follows.

Assume $\mu$ is a probability measure supported on a compact metric space $(X, d)$. The local dimension of $\mu$ at $x$ is

$$
\operatorname{dim}_{\text {loc }}(\mu, x):=\lim _{r \rightarrow 0^{+}} \frac{\log \mu(B(x, r))}{\log r},
$$

if the limit exists. For $\alpha \in \mathbb{R}$, define the level set as

$$
E(\mu, \alpha):=\left\{x \in \operatorname{supp}(\mu): \operatorname{dim}_{\mathrm{loc}}(\mu, x)=\alpha\right\} .
$$

The multifractal analysis is to determine these $\alpha$ such that $E(\mu, \alpha) \neq \varnothing$ and compute the dimension of $E(\mu, \alpha)$ which is called dimension spectra. In many classical cases, the dimension spectra is related to the so-called "free energy" function $\tau_{\mu}$ (also called by $L^{q}$-spectrum), which is defined as follows:

$$
\tau_{\mu}(s)=\lim _{r \rightarrow 0} \frac{\log \sup \left\{\sum_{i}\left(\mu\left(B_{i}\right)\right)^{s}\right\}}{\log r},
$$

where the supremum is taken over all families of disjoint closed balls $B_{i}=B\left(x_{i}, r\right)$ of radius $r$ with centers in $\operatorname{supp}(\mu)$. Of course it is only meaningful when the limit exists. We will say that the multifractal formalism is valid if

$$
\operatorname{dim}_{H} E(\mu, \alpha)=\tau_{\mu}^{*}(\alpha),
$$

where $f^{*}$ denotes the Legendre transform (i.e. $\left.f^{*}(\alpha)=\inf _{q \in \mathbb{R}}\{\alpha q-f(q)\}\right)$ and a negative dimension means that the set is empty.

For many classical systems, such as Cookie-Cutter systems with a Gibbs measure [13], subshift of finite type with a weak Gibbs measure [10], self-similar set with a self-similar measure [9],random weak Gibbs measure [16] and also [5, 6, 8, 15], the multifractal formalism is known to hold. On the other hand, it is also known that for some "bad" measure, the multifractal formalism does not hold. In [3], the authors construct a measure 
such that the multifractal formalism is nowhere valid. The breakdown of multifractal formalism is attributed to the fact that $\tau_{\mu}$ does not eixst.

When $\tau_{\mu}$ does not exist, one can define

$$
\underline{\tau}_{\mu}(s)=\liminf _{r \rightarrow 0} \frac{\log \sup \left\{\sum_{i}\left(\mu\left(B_{i}\right)\right)^{s}\right\}}{\log r}, \text { and } \bar{\tau}_{\mu}(s)=\limsup _{r \rightarrow 0} \frac{\log \sup \left\{\sum_{i}\left(\mu\left(B_{i}\right)\right)^{s}\right\}}{\log r}
$$

respectively.

In [4], the authors provide an example of a measure on the interval $[0,1]$ for which the functions $\underline{\tau}_{\mu}$ and $\bar{\tau}_{\mu}$ differ and the Hausdorff dimensions of the sets $E(\mu, \alpha)$ are given by the Legendre transform of $\bar{\tau}_{\mu}$, and their packing dimensions by the Legendre transform of $\underline{\tau}_{\mu}$ on a subset of the admissible interval. [14] intensifies [4] such that the function $\underline{\tau}_{\mu}$ and $\bar{\tau}_{\mu}$ can be real analytic.

In [12], the author studies the density of states measure of certain Sturm Hamiltonians. He shows that such class of measures is always exact upper and lower dimensional. Here, we call a measure $\mu$ is exact upper dimensional if $\mu$-almost every point $x$, $\overline{\operatorname{dim}}_{\text {loc }}(\mu, x)=C_{1}$ for some constant $C_{1}$, also we call a measure $\mu$ is exact lower dimensional if $\mu$-almost every point $x, \operatorname{dim}_{\mathrm{loc}}(\mu, x)=C_{2}$ for some constant $C_{2}$. His example suggests the possibility that the local dimension might do not exist for any point in the support. This motivates our construction of the present paper. Indeed, our model can serve as a toy model of it, but our results may throw light on its multifractal analysis.

When the local dimension does not exist, we can define the lower and upper local dimensions of $\mu$, i.e.

$$
\underline{\operatorname{dim}}_{\mathrm{loc}}(\mu, x)=\liminf _{r \rightarrow 0^{+}} \frac{\log \mu(B(x, r))}{\log r} \text { and } \overline{\operatorname{dim}}_{\mathrm{loc}}(\mu, x)=\limsup _{r \rightarrow 0^{+}} \frac{\log \mu(B(x, r))}{\log r} .
$$

For $\alpha \in \mathbb{R}$, we define

$$
\begin{aligned}
& \underline{E}(\mu, \alpha)=\left\{x \in \operatorname{supp}(\mu): \underline{\operatorname{dim}}_{\mathrm{loc}}(\mu, x)=\alpha\right\}, \\
& \bar{E}(\mu, \alpha)=\left\{x \in \operatorname{supp}(\mu): \overline{\operatorname{dim}}_{\mathrm{loc}}(\mu, x)=\alpha\right\} .
\end{aligned}
$$

Then we have

$$
E(\mu, \alpha)=\underline{E}(\mu, \alpha) \cap \bar{E}(\mu, \alpha) .
$$

We can further decompose $\underline{E}(\mu, \alpha)$ and $\bar{E}(\mu, \alpha)$ in the following way. For any $\alpha^{\prime} \geq \alpha$, define

$$
E\left(\mu, \alpha, \alpha^{\prime}\right):=\left\{x \in \operatorname{supp}(\mu): \underline{\operatorname{dim}}_{\mathrm{loc}}(\mu, x)=\alpha, \overline{\operatorname{dim}}_{\mathrm{loc}}(\mu, x)=\alpha^{\prime}\right\},
$$

Then we have $E\left(\mu, \alpha, \alpha^{\prime}\right)=\underline{E}(\mu, \alpha) \cap \bar{E}\left(\mu, \alpha^{\prime}\right)$ and

$$
E(\mu, \alpha)=\bigcup_{\alpha^{\prime} \geq \alpha} E\left(\mu, \alpha, \alpha^{\prime}\right), \quad \bar{E}\left(\mu, \alpha^{\prime}\right)=\bigcup_{\alpha \leq \alpha^{\prime}} E\left(\mu, \alpha, \alpha^{\prime}\right) .
$$

In this paper, we will construct a class of Moran measures such that the free energy function does not exist, and that the local dimension does not exist for each point in its support. Then, we will compute the dimensions of $\underline{E}(\mu, \alpha), \bar{E}(\mu, \alpha)$ and $E\left(\mu, \alpha, \alpha^{\prime}\right)$ and indicate that some generalized multifractal formalism holds.

The paper is organized as follows. In section 2, we present our model and state main results. Section 3 provides the basic properties for a more general model which will be useful in the section 4 where we prove our results. 


\section{THE CONSTRUCTION AND MAIN RESUlTS}

Let $\mathcal{A}=\{0,1\}$ and $\mathcal{A}^{*}$ be the set of all finite words on the alphabet $\mathcal{A}$.

Fix two real numbers $A, B$ with $A>B>2$. Let $\mathcal{N}:=\left\{N_{i}\right\}_{i \in \mathbb{N}}$ be an increasing sequence of integers. Define a set $X(A, B, \mathcal{N})$ as follows.

Step 1: Let $I=[0,1]$. For $n=1$, define: $I_{0}=[0,1 / A] \subset I$, and $I_{1}=[1-1 / A, 1] \subset I$.

Step 2: For $n \in \mathbb{N}$ with $n>1$, we assume that for all $w \in \mathcal{A}^{n-1}$, the set $I_{w}$ has been defined. Let $x_{w}$ be the left endpoint of $I_{w}$.

- If $N_{2 i}<n \leq N_{2 i+1}$ for some $i \in \mathbb{N}$, define $I_{w * 0}=\left[x_{w}, x_{w}+\frac{\left|I_{w}\right|}{A}\right]$ and $I_{w * 1}=$ $\left[x_{w}+\left|I_{w}\right|-\frac{\left|I_{w}\right|}{A}, x_{w}+\left|I_{w}\right|\right]$.

- If $N_{2 i+1}<n \leq N_{2 i+2}$ for some $i \in \mathbb{N}$, define $I_{w * 0}=\left[x_{w}, x_{w}+\frac{\left|I_{w}\right|}{B}\right]$ and $I_{w * 1}=\left[x_{w}+\left|I_{w}\right|-\frac{\left|I_{w}\right|}{B}, x_{w}+\left|I_{w}\right|\right]$.

Step 3: Define $X(A, B, \mathcal{N})=\cap_{n \in \mathbb{N}} \cup_{w \in \mathcal{A}^{n}} I_{w}$.

Given two real numbers $p, q$ with $0<p, q \leq 1 / 2$, we will distribute a probability measure $\mu_{(p, q, \mathcal{N})}$ on $X(A, B, \mathcal{N})$ as follows:

Step 1: Let $\mu_{(p, q, \mathcal{N})}(I)=1$. For $n=1$, define: $\mu_{(p, q, \mathcal{N})}\left(I_{0}\right)=p$ and $\mu_{(p, q, \mathcal{N})}\left(I_{1}\right)=1-p$

Step 2: For $n \in \mathbb{N}$ with $n>1$, we assume that for all $w \in \mathcal{A}^{n-1}$, the set $\mu_{(p, q, \mathcal{N})}\left(I_{w}\right)$ has been defined.

- If $N_{2 i}<n \leq N_{2 i+1}$ for some $i \in \mathbb{N}$, define $\mu_{(p, q, \mathcal{N})}\left(I_{w * 0}\right)=p \mu_{(p, q, \mathcal{N})}\left(I_{w}\right)$ and $\mu_{(p, q, \mathcal{N})}\left(I_{w * 1}\right)=(1-p) \mu_{(p, q, \mathcal{N})}\left(I_{w}\right)$.

- If $N_{2 i+1}<n \leq N_{2 i+2}$ for some $i \in \mathbb{N}$, define $\mu_{(p, q, \mathcal{N})}\left(I_{w * 0}\right)=q \mu_{(p, q, \mathcal{N})}\left(I_{w}\right)$ and $\mu_{(p, q, \mathcal{N})}\left(I_{w * 1}\right)=(1-q) \mu_{(p, q, \mathcal{N})}\left(I_{w}\right)$.

Step 3: Such a set function $\mu_{(p, q, \mathcal{N})}$ defined on $\cup_{n \in \mathbb{N}} \mathcal{A}^{n}$ can be extended to a probability measure on the whole $\sigma$-algebra by measure extension theorem. We still denote the measure by $\mu_{(p, q, \mathcal{N})}$.

In this paper, we always make the following assumption:

$$
\lim _{i \rightarrow \infty} \frac{N_{i+1}}{N_{i}}=\infty \text { and }-\frac{\log p}{\log A}<-\frac{\log (1-q)}{\log B} .
$$

Furthermore, if there is no conflict we always denote $X:=X(A, B, \mathcal{N})$ and $\mu:=\mu_{(p, q, \mathcal{N})}$.

Given $0<p, \tilde{p}<1$, define the mixed entropy function

$$
H(\tilde{p}, p):=-\tilde{p} \log p-(1-\tilde{p}) \log (1-p) .
$$

We also write $H(p)=H(p, p)$.

Theorem 2.1. (1) For each point $x \in X=\operatorname{supp}(\mu)$, one has

$$
-\frac{\log (1-p)}{\log A} \leq \underline{\operatorname{dim}}_{\operatorname{loc}}(\mu, x) \leq-\frac{\log p}{\log A}
$$

and

$$
-\frac{\log (1-q)}{\log B} \leq \overline{\operatorname{dim}}_{\mathrm{loc}}(\mu, x) \leq-\frac{\log q}{\log B} .
$$

(2) $\underline{\operatorname{dim}}_{\mathrm{loc}}(\mu, x)<\overline{\operatorname{dim}}_{\mathrm{loc}}(\mu, x)$ for all $x \in X=\operatorname{supp}(\mu)$.

(3) For $\mu$-almost every $x \in X=\operatorname{supp}(\mu)$, one has

$$
{\operatorname{dim}_{\mathrm{loc}}}(\mu, x)=\frac{H(p)}{\log A}, \text { and } \overline{\operatorname{dim}}_{\mathrm{loc}}(\mu, x)=\frac{H(q)}{\log B} .
$$


(4) $\operatorname{dim}_{H} X=\frac{\log 2}{\log A}<\operatorname{dim}_{P} X=\frac{\log 2}{\log B}$.

Remark 2.2. (i) If $p=1 / 2$, then for any $x \in X, \underline{\operatorname{dim}}_{\text {loc }}(\mu, x)=\log 2 / \log A$, hence there is no multifractal analysis for $\underline{E}(\mu, \alpha)$, since $\underline{E}(\mu, \log 2 / \log A)=X$. By the same reason, if $q=1 / 2$, there is no multifractal analysis for $\bar{E}(\mu, \alpha)$.

(ii) The assumption $B>2$ is to ensure that the strong separation condition hold. Using this property, it is equivalent to consider balls and cylinders.

(iii) The second inequality of (1) ensures item 2 of Theorem 2.1, so that this model can provide an example of measure such that the local dimension does not exist for each point in its support. Such nontrivial examples have not been given before to the author's best knowledge.

(iv) (3) is kind of Young's dimension formula.

Next we study the dimensions of the level sets.

Define the function

$$
\begin{aligned}
\beta_{1}: & \mathbb{R} \rightarrow \mathbb{R} \\
s & \mapsto-\frac{\log \left(p^{s}+(1-p)^{s}\right)}{\log A},
\end{aligned}
$$

then

$$
\beta_{1}^{\prime}(+\infty)=\frac{H(0, p)}{\log A} \leq \beta_{1}^{\prime}(1)=\frac{H(p, p)}{\log A} \leq \beta_{1}^{\prime}(0)=\frac{H(1 / 2, p)}{\log A} \leq \beta_{1}^{\prime}(-\infty)=\frac{H(1, p)}{\log A} .
$$

and define $\tilde{\beta}_{1}$ which is a revised function of $\beta_{1}$ (see figure 1 ,

$$
\tilde{\beta}_{1}(s)= \begin{cases}\beta_{1}(s) & s \in(-\infty, 0] \cup[1,+\infty), \\ (1-s) \beta_{1}(0)+s \beta_{1}(1) & s \in(0,1) .\end{cases}
$$

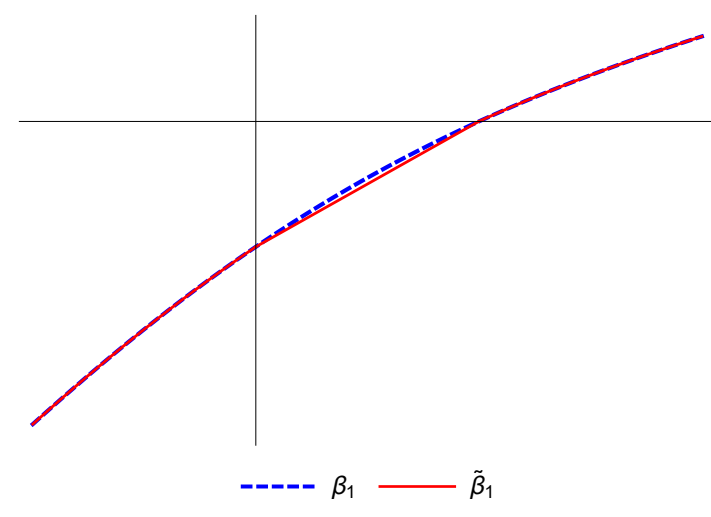

FIGURE 1.

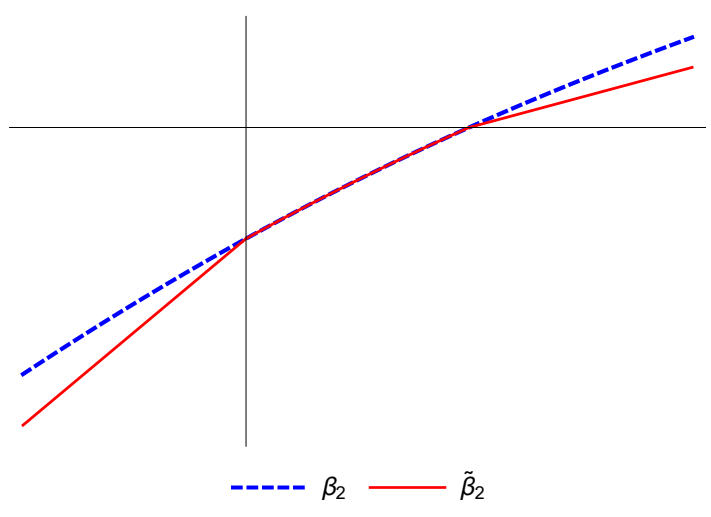

FIGURE 2.

Also, define

$$
\begin{aligned}
\beta_{2}: & \mathbb{R} \rightarrow \mathbb{R} \\
s & \mapsto-\frac{\log \left(q^{s}+(1-q)^{s}\right)}{\log B},
\end{aligned}
$$


then

$$
\beta_{2}^{\prime}(+\infty)=\frac{H(0, q)}{\log B} \leq \beta_{2}^{\prime}(1)=\frac{H(q, q)}{\log B} \leq \beta_{2}^{\prime}(0)=\frac{H(1 / 2, q)}{\log B} \leq \beta_{2}^{\prime}(-\infty)=\frac{H(1, q)}{\log B} .
$$

and define the revised function of $\beta_{2}$ (see figure 2)

$$
\tilde{\beta}_{2}(s)= \begin{cases}\beta_{2}(0)+s \beta_{2}^{\prime}(0) & s \in(-\infty, 0), \\ \beta_{2}(s) & s \in[0,1], \\ \beta_{2}(1)+\beta_{2}^{\prime}(1)(s-1) & s \in(1,+\infty) .\end{cases}
$$

The two functions $\beta_{1}, \beta_{2}$ can be seen as the $L^{q}$-spectrum if we just use the data $(A, p)$ or $(B, q)$.

Theorem 2.3. (1) If $\alpha \notin\left[\beta_{1}^{\prime}(+\infty), \beta_{1}^{\prime}(-\infty)\right], \underline{E}(\mu, \alpha)=\varnothing$.

If $\alpha \notin\left[\beta_{2}^{\prime}(+\infty), \beta_{2}^{\prime}(-\infty)\right], \bar{E}(\mu, \alpha)=\varnothing$.

(2) For $\alpha \in\left[\beta_{1}^{\prime}(+\infty), \beta_{1}^{\prime}(-\infty)\right]$, we have

$$
\operatorname{dim}_{H} \underline{E}(\mu, \alpha)=\tilde{\beta}_{1}^{*}(\alpha) \text { and } \operatorname{dim}_{P} \underline{E}(\mu, \alpha)=\frac{\log 2}{\log B} .
$$

(3) For $\alpha \in\left[\beta_{2}^{\prime}(+\infty), \beta_{2}^{\prime}(-\infty)\right]$, we have

$$
\operatorname{dim}_{H} \bar{E}(\mu, \alpha)=\min \left\{\frac{\log 2}{\log A}, \beta_{2}^{*}(\alpha)\right\}
$$

and

$$
\operatorname{dim}_{P} \bar{E}(\mu, \alpha)=\tilde{\beta}_{2}^{*}(\alpha)
$$

Here we draw figure 3 and give some remarks to illustrate theorem 2.3 .

Remark 2.4. (i) We will compute the explicit formula of the Hausdorff dimension spectrum as

$$
\operatorname{dim}_{H} \underline{E}(\mu, \alpha)= \begin{cases}\beta_{1}^{*}(\alpha) & \alpha \in\left[\beta_{1}^{\prime}(+\infty), \beta_{1}^{\prime}(1)\right) \cup\left[\beta_{1}^{\prime}(0), \beta_{1}^{\prime}(-\infty)\right], \\ \min \left\{\alpha, \frac{\log 2}{\log A}\right) & \alpha \in\left[\beta_{1}^{\prime}(1), \beta_{1}^{\prime}(0)\right)\end{cases}
$$

which turns out to be $\tilde{\beta}_{1}^{*}(\alpha)$.

(ii) We will also compute the explicit formula of the Hausdorff dimension spectrum as

$$
\operatorname{dim}_{P} \bar{E}(\mu, \alpha)= \begin{cases}\alpha & \alpha \in\left[\beta_{2}^{\prime}(+\infty), \beta_{2}^{\prime}(1)\right), \\ \beta_{2}^{*}(\alpha) & \alpha \in\left[\beta_{2}^{\prime}(1), \beta_{2}^{\prime}(0)\right) \\ \frac{\log 2}{\log B} & \alpha \in\left[\beta_{2}^{\prime}(0), \beta_{2}^{\prime}(-\infty)\right] .\end{cases}
$$

which turns out to be $\tilde{\beta}_{2}^{*}(\alpha)$.

At last, we compute the dimension of $E\left(\mu, \alpha, \alpha^{\prime}\right)$.

Theorem 2.5. Assume $\left(\alpha, \alpha^{\prime}\right) \in\left[\beta_{1}^{\prime}(+\infty), \beta_{1}^{\prime}(-\infty)\right] \times\left[\beta_{2}^{\prime}(+\infty), \beta_{2}^{\prime}(-\infty)\right]$.

(1) we have

- if $\left(\alpha, \alpha^{\prime}\right) \notin\left[\beta_{1}^{\prime}(1), \beta_{1}^{\prime}(0)\right] \times\left[\beta_{2}^{\prime}(+\infty), \beta_{2}^{\prime}(1)\right)$, i.e. $\left(\alpha, \alpha^{\prime}\right)$ in domain I (the Blue part) of figure 4

$$
\operatorname{dim}_{H}\left(E\left(\mu, \alpha, \alpha^{\prime}\right)\right)=\min \left\{\operatorname{dim}_{H} \underline{E}(\mu, \alpha), \operatorname{dim}_{H} \bar{E}\left(\mu, \alpha^{\prime}\right)\right\} .
$$




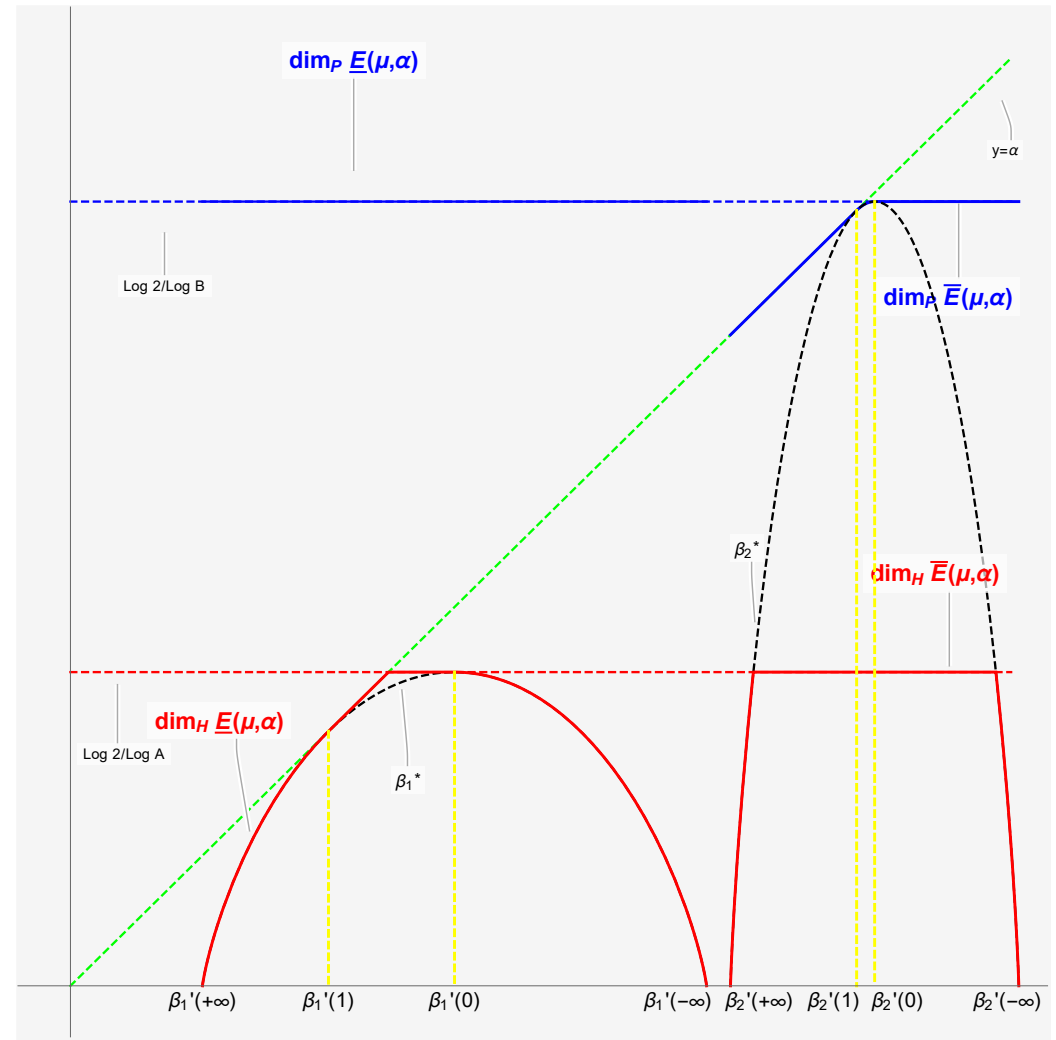

FIGURE 3. The Hausdorff and packing dimension for $\underline{E}(\mu, \alpha)$ and $\bar{E}(\mu, \alpha)$

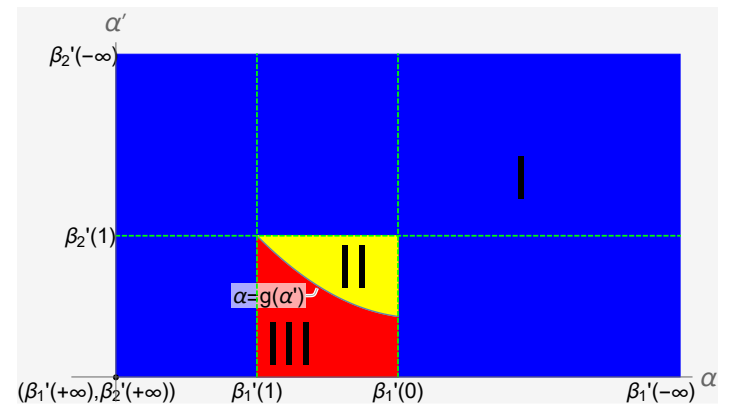

FIGURE 4. Domain for Hausdorff dimension

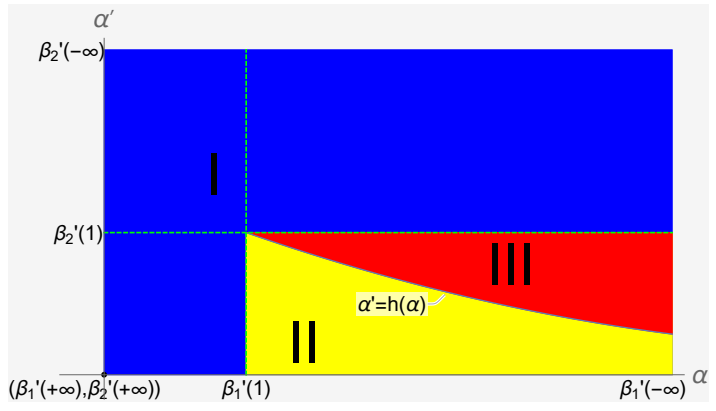

FIGURE 5. Domain for Packing dimension

- if $\alpha^{\prime} \in\left[\beta_{2}^{\prime}(+\infty), \beta_{2}^{\prime}(1)\right)$ with $g\left(\alpha^{\prime}\right) \leq \beta_{1}^{\prime}(0)$, where we take the tangent to the graph $\beta_{1}^{*}$ passing through the point $\left(\alpha^{\prime}, \beta_{2}^{*}\left(\alpha^{\prime}\right)\right)$ and denote the point of tangency by $\left(g\left(\alpha^{\prime}\right), \beta_{1}^{*}\left(g\left(\alpha^{\prime}\right)\right)\right)$. (One can refer figure 6 for the definition of the function $\left.g.\right)$ For $\alpha \in\left[g\left(\alpha^{\prime}\right), \beta_{1}^{\prime}(0)\right)$, i.e. $\left(\alpha, \alpha^{\prime}\right)$ in domain II (the yellow part) of figure 4

$$
\operatorname{dim}_{H}\left(E\left(\mu, \alpha, \alpha^{\prime}\right)\right)=\min \left\{\frac{\log 2}{\log A}, s_{1} \alpha-\beta_{1}\left(s_{1}\right)\right\},
$$

where $s_{1} \in[0,1]$ satisfies $\beta_{1}^{\prime}\left(s_{1}\right)=g\left(\alpha^{\prime}\right)$. 
- otherwise, i.e. $\left(\alpha, \alpha^{\prime}\right)$ in domain III (the red part) of figure 4

$$
\operatorname{dim}_{H}\left(E\left(\mu, \alpha, \alpha^{\prime}\right)\right)=\min \left\{\beta_{1}^{*}(\alpha), \beta_{2}^{*}\left(\alpha^{\prime}\right)\right\} .
$$

(2) For $\left(\alpha, \alpha^{\prime}\right) \in\left[\beta_{1}^{\prime}(+\infty), \beta_{1}^{\prime}(-\infty)\right] \times\left[\beta_{2}^{\prime}(+\infty), \beta_{2}^{\prime}(-\infty)\right]$, we have

- if $\left(\alpha, \alpha^{\prime}\right) \notin\left[\beta_{1}^{\prime}(1), \beta_{1}^{\prime}(-\infty)\right] \times\left[\beta_{2}^{\prime}(+\infty), \beta_{2}^{\prime}(1)\right),\left(\alpha, \alpha^{\prime}\right)$ in domain I (the blue part) of figure 5

$$
\operatorname{dim}_{P}\left(E\left(\mu, \alpha, \alpha^{\prime}\right)\right)=\operatorname{dim}_{P} \bar{E}\left(\mu, \alpha^{\prime}\right) .
$$

- if $\alpha \in\left[\beta_{1}^{\prime}(1), \beta_{1}^{\prime}(-\infty)\right]$ and $\alpha^{\prime} \in\left[\beta_{2}^{\prime}(+\infty), h(\alpha)\right)$. Here, we take the tangent to the graph $\beta_{2}^{*}$ passing through the point $\left(\alpha, \beta_{1}^{*}(\alpha)\right)$ and denote the point of tangency by $\left(h(\alpha), \beta_{2}^{*}(h(\alpha))\right)$. (One can refer figure 7 for the definition of the function $h$.) Then take $s_{2} \in(1,+\infty]$ such that $\beta_{2}^{\prime}\left(s_{2}\right)=h(\alpha)$. In this case, $\left(\alpha, \alpha^{\prime}\right)$ in domain II (the Yellow part) of figure 5

$$
\operatorname{dim}_{P}\left(E\left(\mu, \alpha, \alpha^{\prime}\right)\right)=s_{2} \alpha^{\prime}-\beta_{2}\left(s_{2}\right) .
$$

- otherwise, $\left(\alpha, \alpha^{\prime}\right)$ in domain III (the red part) of figure 5

$$
\operatorname{dim}_{P}\left(E\left(\mu, \alpha, \alpha^{\prime}\right)\right)=\beta_{2}^{*}\left(\alpha^{\prime}\right) .
$$

We now draw figure 6 (the red line) for $\operatorname{dim}_{H}\left(E\left(\mu, \alpha, \alpha^{\prime}\right)\right)$ when $\alpha \in\left[\beta_{1}^{\prime}(1), \beta_{1}^{\prime}(0)\right]$ for a fixed $\alpha^{\prime} \in\left[\beta_{2}^{\prime}(+\infty), \beta_{2}^{\prime}(1)\right)$ with $g\left(\alpha^{\prime}\right) \leq \beta_{1}^{\prime}(0)$ and figure 7 (the red line) for $\operatorname{dim}_{P}\left(E\left(\mu, \alpha, \alpha^{\prime}\right)\right)$ when $\alpha^{\prime} \in\left[\beta_{2}^{\prime}(+\infty), \beta_{2}^{\prime}(1)\right]$ with a fixed $\alpha \in\left(\beta_{2}^{\prime}(1), \beta_{2}^{\prime}(-\infty)\right]$ to illustrate theorem 2.5 .

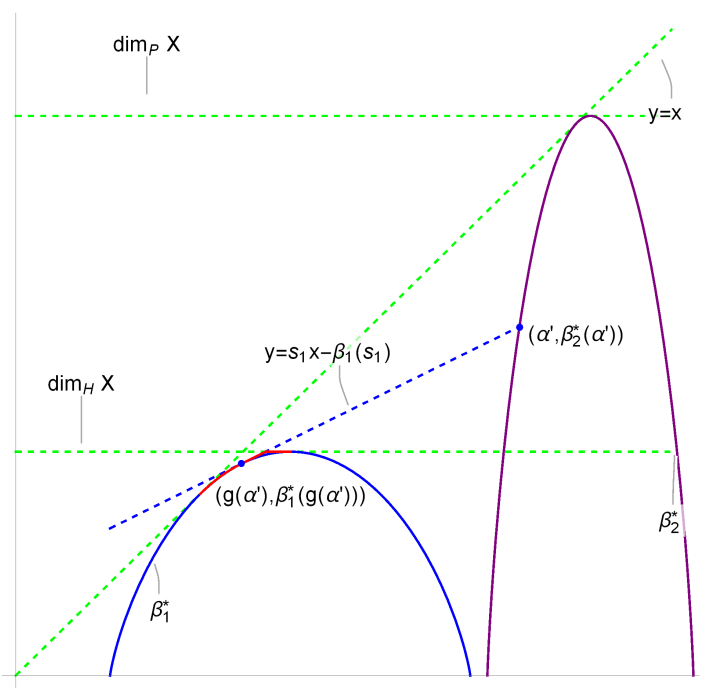

FigURE 6. $\operatorname{dim}_{H}\left(E\left(\mu, \cdot \alpha^{\prime}\right)\right)$

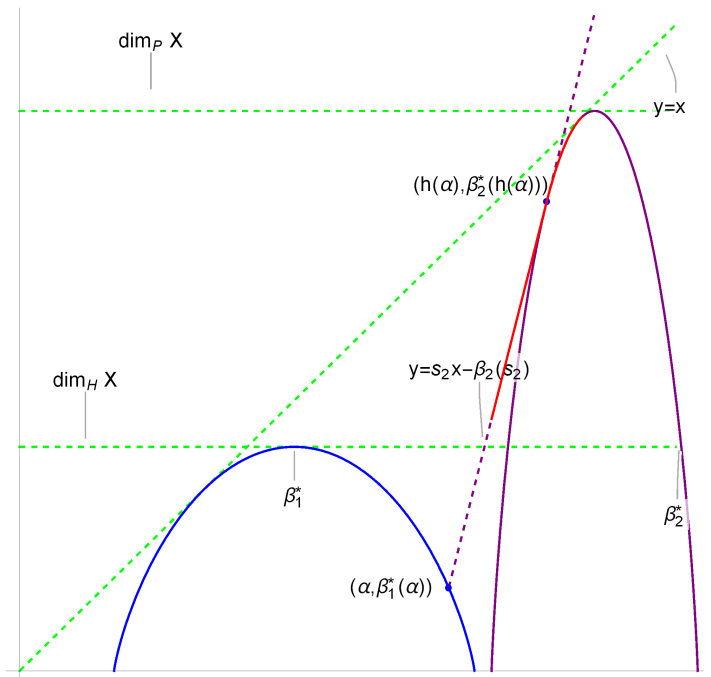

FigURE 7. $\operatorname{dim}_{P}(E(\mu, \alpha, \cdot))$

Let us give some remarks of this paper.

(1) We refer the reader to [16], where the author deals with the random weak Gibbs measures. Under some natural conditions on transitivity and conformality, almost surely, it gives a full description of the multifractal analysis. But, unfortunately, it could not give any information for a sample in the exceptional set with measure 0 . A crucial problem is to deal with such samples. Is this still true? Is the level set 
with local dimension always nonempty? What can their multifractal behavior be? Our model provides a non-trivial sample with a full description of the multifractal analysis.

(2) The inequality in (1) is not critical for the calculation of the dimension of level sets. Even if we do not have the inequality, our method can also be effective. In this sense, we can get the full results for the example in [4]. We focus this model to avoid discussing too many situations.

(3) We need to emphasize that we are dealing the level set with respect to upper and lower local dimensions under the nonexistence of the "free energy" function. The lower bounds for $\operatorname{dim}_{H} \underline{E}(\mu, \alpha)$ when $\alpha \in\left[\beta_{1}^{\prime}(1), \beta_{1}^{\prime}(0)\right)$ and $\operatorname{dim}_{P} \bar{E}(\mu, \alpha)$ when $\alpha \in\left[\beta_{2}^{\prime}(+\infty), \beta_{1}^{\prime}(1)\right)$ are new phenomena and have not been obtained before. For the set $E\left(\mu, \alpha, \alpha^{\prime}\right)$, the upper bounds of the (Hausdorff and packing) dimensions are also new phenomena for the part if those of $E(\mu, \alpha)$ and $\bar{E}(\mu, \alpha)$ could not provide the exact upper bounds.

(4) The strong law of large numbers [7, Theorem 5.4.1] plays an important role in this paper. The classical strong law of large numbers can deal with infinite sequence of independent identically distributed random variables, but in our situation they are not identically distributed.

\section{A GENERAL MODEL}

We now set a generalization of the model presented in section 2 . Fix a sequence of positive numbers $\left\{A_{n}\right\}_{n \in \mathbb{N}}$ with $A_{n} \geq 2$ for any $n \in \mathbb{N}$.

Now we consider the following construction:

Step 1: Let $I=[0,1]$, for $n=1$, define: $I_{0}=\left[0,1 / A_{1}\right] \subset I$, and $I_{1}=\left[1-1 / A_{1}, 1\right] \subset I$.

Step 2: For $n \in \mathbb{N}$ with $n>1$, we assume that for all $w \in \mathcal{A}^{n-1}$, the set $I_{w}$ have been defined. Let $x_{w}$ be the left endpoint of $I_{w}$. Define $I_{w * 0}=\left[x_{w}, x_{w}+\frac{\left|I_{w}\right|}{A_{n}}\right]$ and $I_{w * 1}=$ $\left[x_{w}+\left|I_{w}\right|-\frac{\left|I_{w}\right|}{A_{n}}, x_{w}+\left|I_{w}\right|\right]$.

Step 3: Define $Y=\cap_{n \in \mathbb{N}} \cup_{w \in \mathcal{A}^{n}} I_{w}$.

Then, fix $a, b \in \mathbb{R}$ such that $0<a \leq b<1$ and a sequence of positive numbers $\left\{p_{n}\right\}_{n \in \mathbb{N}}$ with $a \leq p_{n} \leq b$ for any $n \in \mathbb{N}$.

Now we distribute a measure on $Y$ :

Step 1: $v(I)=1$. For $n=1$, define: $v\left(I_{0}\right)=p_{1}$ and $v\left(I_{1}\right)=1-p_{1}$.

Step 2: For $n \in \mathbb{N}$ with $n>1$, we assume that $v\left(I_{\sigma}\right)$ have been defined for all $\sigma \in \mathcal{A}^{n-1}$. Define $v\left(I_{\sigma * 0}\right)=p_{n} v\left(I_{\sigma}\right)$ and $v\left(I_{\sigma * 1}\right)=\left(1-p_{n}\right) v\left(I_{\sigma}\right)$.

Step 4: We can extend $v$ to be a probability measure on $Y$ by measure extension theorem, then $\operatorname{supp}(v)=Y$.

Define $d(v, x, n)=\frac{\log v\left(I_{n}(x)\right)}{\log \left|I_{n}(x)\right|}$, where $I_{n}(x)$ is the cylinder $I_{w}$ with $x \in I_{w}$ and $w=$ $w_{1} w_{2} \cdots w_{n}$ of length $n$.

Now we choose a sequence $\left\{p_{n}^{\prime}\right\}_{n \in \mathbb{N}}$ with $0 \leq p_{n}^{\prime} \leq 1$. We consider the measure $v^{\prime}$ constructed as $v$ with parameters $\left\{p_{n}^{\prime}\right\}_{n \in \mathbb{N}}$ instead of $\left\{p_{n}\right\}_{n \in \mathbb{N}}$.

Now we state a version of strong law of large numbers which will be useful in this paper.

Theorem 3.1. [7. Theorem 5.4.1] Let $\left\{X_{n}\right\}_{n \in \mathbb{N}}$ be a sequence of independent random variables with $\mathbb{E}\left(X_{n}\right)=0$ for every $n \in \mathbb{N}$, and $\left\{a_{n}\right\}_{n \in \mathbb{N}}$ positive and increasing to $+\infty$. If there exists $a$ function $\phi$ such that $\phi$ is positive, even and continuous on $\mathbb{R}$ such that 
- when $|x|$ increases, $\frac{\phi(x)}{|x|}$ does not decrease and $\frac{\phi(x)}{x^{2}}$ does not increase,

- $\sum_{n} \frac{\mathbb{E}\left(\phi\left(X_{n}\right)\right)}{\phi\left(a_{n}\right)}<+\infty$.

Then $\sum_{n} \frac{X_{n}}{a_{n}}$ converge almost everywhere. Furthermore, $\frac{1}{a_{n}} \sum_{i=1}^{n} X_{i}$ converges to 0 as $n \rightarrow \infty$ almost everywhere.

From theorem 3.1. we can get the following lemma.

Lemma 3.2. 1) For $v^{\prime}$-almost every $x \in Y=\operatorname{supp}(v)$, one has

$$
\liminf _{n \rightarrow \infty} d(v, x, n)=\liminf _{n \rightarrow \infty} \frac{\sum_{k=1}^{n} x_{k}}{\sum_{k=1}^{n} b_{k}}
$$

and

$$
\limsup _{n \rightarrow \infty} d(v, x, n)=\limsup _{n \rightarrow \infty} \frac{\sum_{k=1}^{n} x_{k}}{\sum_{k=1}^{n} b_{k}} .
$$

where $x_{k}=-p_{k}^{\prime} \log p_{k}-\left(1-p_{k}^{\prime}\right) \log \left(1-p_{k}\right)$.

2) For $v^{\prime}$-almost every $x \in \operatorname{supp}\left(v^{\prime}\right) \subset Y$, one has

$$
\liminf _{n \rightarrow \infty} d\left(v^{\prime}, x, n\right)=\liminf _{n \rightarrow \infty} \frac{\sum_{k=1}^{n} y_{k}}{\sum_{k=1}^{n} b_{k}}
$$

and

$$
\limsup _{n \rightarrow \infty} d\left(v^{\prime}, x, n\right)=\limsup _{n \rightarrow \infty} \frac{\sum_{k=1}^{n} y_{k}}{\sum_{k=1}^{n} b_{k}}
$$

where $y_{k}=-p_{k}^{\prime} \log p_{k}^{\prime}-\left(1-p_{k}^{\prime}\right) \log \left(1-p_{k}^{\prime}\right)$.

Proof. We just fix our attention on item 1), the next one is the same.

Define the random variable $X_{k}$ as

$$
X_{k}(x)= \begin{cases}-\log p_{k} & I_{k}(x)=I_{w} \text { with } w_{k}=0, \\ -\log \left(1-p_{k}\right) & I_{k}(x)=I_{w} \text { with } w_{k}=1\end{cases}
$$

Then, for the measure $v^{\prime},\left\{X_{k}-\mathbb{E}\left(X_{k}\right)\right\}_{n \in \mathbb{N}}$ is a sequence of independent random variables with $\mathbb{E}\left(X_{k}-\mathbb{E}\left(X_{k}\right)\right)=0$

Define $b_{k}=\log A_{k}$, we replace the $X_{n}, a_{n}$ and $\phi$ in theorem 3.1 by $X_{n}-\mathbb{E}\left(X_{n}\right), \sum_{k=1}^{n} b_{k}$ and the function $s \mapsto s^{2}$, we can conclude for $v^{\prime}$-almost every $x \in Y$

$$
\lim _{n \rightarrow \infty} \frac{\sum_{k=1}^{n}\left(X_{k}(x)-\mathbb{E}\left(X_{k}\right)\right)}{\sum_{k=1}^{n} b_{k}}=0 .
$$

So that

$\liminf _{n \rightarrow \infty} \frac{\sum_{k=1}^{n} X_{k}(x)}{\sum_{k=1}^{n} b_{k}}=\liminf _{n \rightarrow \infty} \frac{\sum_{k=1}^{n} \mathbb{E}\left(X_{k}\right)}{\sum_{k=1}^{n} b_{k}}$ and $\limsup _{n \rightarrow \infty} \frac{\sum_{k=1}^{n} X_{k}(x)}{\sum_{k=1}^{n} b_{k}}=\limsup _{n \rightarrow \infty} \frac{\sum_{k=1}^{n} \mathbb{E}\left(X_{k}\right)}{\sum_{k=1}^{n} b_{k}}$.

Item 1) valid if we notice that $d(v, x, n)=\frac{\sum_{k=1}^{n} X_{k}(x)}{\sum_{k=1}^{n} b_{k}}$ and $\mathbb{E}\left(X_{k}\right)=x_{k}$.

Now if we assume that $2<\underline{A}:=\inf \left\{A_{n}: n \in \mathbb{N}\right\}$, then we know that the strong separation condition holds. We now also assume $\bar{A}:=\sup \left\{A_{n}: n \in \mathbb{N}\right\}<+\infty$. We can get the following lemma by the same method as in [8, Corollary 4.3].

Lemma 3.3. For any ball $B(x, r)$ with $x \in Y$ and $r>0$, we choose the smallest $n \in \mathbb{N}$ and the largest $n^{\prime} \in \mathbb{N}$ such that $I_{n}(x) \subset B(x, r)$ and $(B(x, r) \cap Y) \subset I_{n^{\prime}}(x)$. Then we have

$$
\frac{r}{\bar{A}} \leq \frac{r}{A_{n}} \leq\left|I_{n}(x)\right| \leq 2 r \text { and }\left|I_{n^{\prime}}(x)\right| \leq \frac{2\left(A_{n^{\prime}+1}\right) r}{A_{n^{\prime}+1}-2} \leq \frac{2 \underline{A} r}{\underline{A}-2}
$$


The proof of lemma 3.3 is obvious if we notice that $\left|I_{n-1}(x)\right| \geq r$ and the $\left(n^{\prime}+1\right)$-th gap in $I_{n^{\prime}}(x)$ is a subset of $B(x, r)$.

Lemma 3.2 and 3.3 can deduce the following lemma.

Lemma 3.4. If we assume that $2<\underline{A} \leq \bar{A}<+\infty$,

1) for each point $x \in Y=\operatorname{supp}(v)$,

$$
\underline{\operatorname{dim}}_{\mathrm{loc}}\left(v^{\prime}, x\right)=\liminf _{n \rightarrow \infty} d\left(v^{\prime}, x, n\right) \text { and } \overline{\operatorname{dim}}_{\mathrm{loc}}\left(v^{\prime}, x\right)=\limsup _{n \rightarrow \infty} d\left(v^{\prime}, x, n\right) .
$$

2) the measure $v^{\prime}$ is exact upper and lower dimensional.

\section{PROOF OF THE RESUlts}

Now we return to the model defined in section 2 .

4.1. Proof of Theorem 2.1. In fact we just need to notice $\lim _{i \rightarrow \infty} \frac{N_{i+1}}{N_{i}}=\infty$, lemma 3.2 and 3.4 . The proof is the following.

Proof. (1) We just need to notice that

$$
\begin{gathered}
-\frac{\log (1-p)}{\log A} \leq d(v, x, n) \leq-\frac{\log q}{\log B^{\prime}} \\
\liminf _{i \rightarrow \infty} d\left(v, x, N_{2 i+1}\right) \leq-\frac{\log p}{\log A}
\end{gathered}
$$

and

$$
\limsup _{i \rightarrow \infty} d\left(v, x, N_{2 i}\right) \geq-\frac{\log (1-q)}{\log B} .
$$

The results can be obtained from lemma 3.4 .

(2) It is from inequality (1) and the previous item.

(3) It is direct due to the construction and lemma 3.2 and 3.4

(4) We just need to distribute a mass replacing $p$ and $q$ by $1 / 2$, it is obvious that for all $x \in X, \underline{\operatorname{dim}}_{\mathrm{loc}}(\mu, x)=\frac{\log 2}{\log A}$ and $\overline{\operatorname{dim}}_{\mathrm{loc}}(\mu, x)=\frac{\log 2}{\log B}$. By [8, Proposition 2.3], the results hold.

4.2. Proof of Theorem 2.3. From the construction, since $A>B>2$ and the lengths of cylinders $I_{w}, w \in \mathcal{A}^{n}$ are equal for any $n \in \mathbb{N}$, we can easily obtain the following lemma from lemma 3.3 .

\section{Lemma 4.1.}

$$
\underline{\tau}(s)=\min \left\{\beta_{1}(s), \beta_{2}(s)\right\}
$$

and

$$
\bar{\tau}(s)=\max \left\{\beta_{1}(s), \beta_{2}(s)\right\} .
$$

We also need to introduce the large deviations spectra (see also [1]) which will be useful in our proof. 
Let $\mu$ be a compactly supported positive and finite Borel measure on a metric space. For $0 \leq \alpha \leq \beta \leq+\infty$, the lower and upper large deviations spectra $f_{\mu}^{L D}$ and $\bar{f}_{\mu}^{L D}$ are given by

$$
\begin{aligned}
& f_{\mu}^{L D}(\alpha, \beta)=\lim _{\varepsilon \rightarrow 0} \liminf _{r \rightarrow 0} \frac{\log \sup \#\left\{i: r^{\beta+\varepsilon} \leq \mu\left(B\left(x_{i}, r\right) \leq r^{\alpha-\varepsilon}\right)\right\}}{-\log r}, \\
& \bar{f}_{\mu}^{L D}(\alpha, \beta)=\lim _{\varepsilon \rightarrow 0} \limsup _{r \rightarrow 0} \frac{\log \sup \#\left\{i: r^{\beta+\varepsilon} \leq \mu\left(B\left(x_{i}, r\right) \leq r^{\alpha-\varepsilon}\right)\right\}}{-\log r},
\end{aligned}
$$

where the supremum is taken over all families of disjoint closed balls $B_{i}=B\left(x_{i}, r\right)$ of radius $r$ with centers $x_{i}$ in $\operatorname{supp}(\mu)$.

Now we turn to consider the level sets and prove theorem 2.3 .

For any $\alpha \in\left[\beta_{1}^{\prime}(+\infty), \beta_{1}^{\prime}(-\infty)\right]$, if there exists $s \in \mathbb{R}$ such that $\beta_{1}^{\prime}(s)=\alpha$, we can define the number $A^{\beta_{1}(s)} \cdot p^{s}$, otherwise, $\alpha \in\left\{\beta_{1}^{\prime}(+\infty), \beta_{1}^{\prime}(-\infty)\right\}$, we write $A^{\beta_{1}(+\infty)}$. $p^{+\infty}:=\lim _{s \rightarrow+\infty} A^{\beta_{1}(s)} \cdot p^{s}$ and $A^{\beta_{1}(-\infty)} \cdot p^{-\infty}:=\lim _{s \rightarrow-\infty} A^{\beta_{1}(s)} \cdot p^{s}$. Also, we use the same notation for $\beta_{2}$.

Proof. (1) It is obvious because of theorem 2.1

(2) Now we consider the level set $\underline{E}(\mu, \alpha))$.

- Upper bound for $\operatorname{dim}_{H}(\underline{E}(\mu, \alpha))$ and $\operatorname{dim}_{P}(\underline{E}(\mu, \alpha))$ :

One obvious upper bound is $\operatorname{dim}_{H}(\underline{E}(\mu, \alpha)) \leq \operatorname{dim}_{H} X=\frac{\log 2}{\log A}$ and $\operatorname{dim}_{P}(\underline{E}(\mu, \alpha)) \leq$ $\operatorname{dim}_{P} X=\frac{\log 2}{\log B}$. But it is not sharp for the Hausdorff dimension, so we need the following estimation.

- For $\alpha \in\left[\beta_{1}^{\prime}(+\infty), \beta_{1}^{\prime}(0)\right]$, noticing the result in [1, Proposition $1.3 \&$ inequality (1.5)], we know that

$$
\operatorname{dim}_{H} \underline{E}(\mu, \alpha) \leq \bar{f}_{\mu}^{L D}(\alpha, \alpha) \leq \underline{\tau}^{*}(\alpha)= \begin{cases}\beta_{1}^{*}(\alpha) & \alpha \in\left[\beta_{1}^{\prime}(+\infty), \beta_{1}^{\prime}(1)\right], \\ \alpha & \alpha \in\left[\beta_{1}^{\prime}(1), \beta_{1}^{\prime}(0)\right] .\end{cases}
$$

- For $\alpha \in\left[\beta_{1}^{\prime}(0), \beta_{1}^{\prime}(-\infty)\right]$. Noticing the result in [1, Proposition 1.3], $\operatorname{dim}_{H} \underline{E}(\mu, \alpha) \leq \underline{f}_{\mu}^{L D}(\alpha,+\infty)=: f_{1}(\alpha)$.

We just need to prove that $\beta_{1}(s) \leq\left(f_{1}\right)^{*}(s)$ for $s \in(-\infty, 0]$. If so,

$$
\begin{aligned}
\beta_{1}^{*}(\alpha) & =\inf \left\{s \alpha-\beta_{1}(s)\right\} \\
& =\inf _{s \leq 0}\left\{s \alpha-\beta_{1}(s)\right\} \\
& \geq \inf _{s \leq 0}\left\{s \alpha-\left(f_{1}\right)^{*}(s)\right\} \\
& \geq \inf _{s \leq 0}\left\{s \alpha-\left(s \alpha-f_{1}(\alpha)\right)\right\} \\
& =f_{1}(\alpha),
\end{aligned}
$$

and then $\operatorname{dim}_{H} \underline{E}(\mu, \alpha) \leq \beta_{1}^{*}(\alpha)$.

Now turn to prove $\beta_{1}(s) \leq\left(f_{1}\right)^{*}(s)$ for $s \in(-\infty, 0]$. We will borrow the main idea in [1, Section 5.3], but make it clear that we are dealing with $s \leq 0$. If $\left\{B\left(x_{i}, r\right)\right\}$ is a packing of $\operatorname{supp}(\mu)$ by disjoint balls, we have for any $t \in \mathbb{R}$,

$$
\sum_{i} \mu\left(B\left(x_{i}, r\right)\right)^{s} \geq\left(\#\left\{i: \mu\left(B\left(x_{i}, r\right)\right) \leq r^{t-\epsilon}\right\}\right) r^{s(t-\epsilon)} .
$$


Taking the supremum over the packings, dividing by $\log r$, taking the $\lim$ sup as $r \rightarrow 0^{+}$and then the limit $\epsilon \rightarrow 0^{+}$yields $\beta_{1}(s)=\bar{\tau}(s) \leq$ $s t-f_{1}(t)$ for all $t \in \mathbb{R}$, that is $\beta_{1}(s) \leq\left(f_{1}\right)^{*}(s)$

- Lower bound for $\operatorname{dim}_{H}(\underline{E}(\mu, \alpha))$ and $\operatorname{dim}_{P}(\underline{E}(\mu, \alpha))$ :

For any $\alpha \in\left[\beta_{1}^{\prime}(+\infty), \beta_{1}^{\prime}(-\infty)\right]$, there exists $s \in \mathbb{R} \cup\{+\infty,-\infty\}$ such that $\beta_{2}^{\prime}(s)=\alpha$. Choose

$$
p_{n}^{\prime}= \begin{cases}A^{\beta_{1}(s)} \cdot p^{s} & N_{2 i}<n \leq N_{2 i+1} \text { for some } i \in \mathbb{N}, \\ 1 / 2 & N_{2 i+1}<n \leq N_{2 i+2} \text { for some } i \in \mathbb{N} .\end{cases}
$$

to define $\mu^{\prime}$.

Noticing

$$
\frac{-p^{\prime} \log p-\left(1-p^{\prime}\right) \log (1-p)}{\log A}=\beta_{1}^{\prime}(s)=\alpha,
$$

where $p^{\prime}=: A^{\beta_{1}(s)} \cdot p^{s}$. From lemma 3.2 and 3.4 and the choice of $s$, we get that $\mu^{\prime}(\underline{E}(\mu, \alpha))=1$ and for $\mu^{\prime}$-almost every $x \in X$ one has $\underline{\operatorname{dim}}_{\mathrm{loc}}\left(\mu^{\prime}, x\right)=$ $\frac{-p^{\prime} \log p^{\prime}-\left(1-p^{\prime}\right) \log \left(1-p^{\prime}\right)}{\log A}=s \alpha-\beta_{1}(s)=\beta_{1}^{*}(\alpha)$ and $\overline{\operatorname{dim}}_{\mathrm{loc}}\left(\mu^{\prime}, x\right)=\frac{\log 2}{\log B}$. This gives that

$$
\operatorname{dim}_{H} \underline{E}(\mu, \alpha) \geq \beta_{1}^{*}(\alpha)
$$

and

$$
\operatorname{dim}_{P} \underline{E}(\mu, \alpha) \geq \frac{\log 2}{\log B}
$$

But it is not sharp for $\operatorname{dim}_{H} \underline{E}(\mu, \alpha)$ when $\alpha \in\left(\beta_{1}^{\prime}(1), \beta_{1}^{\prime}(0)\right)$. Now take $\alpha_{1}=$ $\frac{-p \log p-(1-p) \log (1-p)}{\log A}$ and $\alpha_{2}=\frac{-q \log q-(1-q) \log (1-q)}{\log B}$, define

$$
p_{n}^{\prime}= \begin{cases}p & N_{2 i}<n \leq N_{2 i}^{\prime} \text { for some } i \in \mathbb{N} \\ 1 / 2 & N_{2 i}^{\prime}<n \leq N_{2 i+1} \text { for some } i \in \mathbb{N} \\ q & N_{2 i+1}<n \leq N_{2 i+2} \text { for some } i \in \mathbb{N} .\end{cases}
$$

where $N_{2 i}^{\prime}=\min \left\{N_{2 i+1},\left\lfloor\frac{\left(\alpha_{2}-\alpha\right)\left(\sum_{k=0}^{i-1}\left(N_{2 k+2}-N_{2 k+1}\right)\right) \log B}{\left(\alpha-\alpha_{1}\right) \log A}\right\rfloor-\sum_{k=0}^{i-1}\left(N_{2 k}^{\prime}-N_{2 k}\right)\right\}$. This also gives a measure $\mu^{\prime}$.

We use the same notation as in lemma 3.2 and turn to analysis $\frac{\sum_{k=1}^{n} x_{k}}{\sum_{k=1}^{n} b_{k}}$. For any $n \in \mathbb{N}$, we assume that $l_{1}(n)=\#\left\{k \leq n: p_{k}^{\prime}=p\right\}, l_{2}(n)=\#\left\{k \leq n: p_{k}^{\prime}=\right.$ $1 / 2\}$ and $l_{3}(n)=\#\left\{k \leq n: p_{k}^{\prime}=q\right\}$ then $l_{1}(n)+l_{2}(n)+l_{3}(n)=n$. From the choice of $N_{2 i}^{\prime}$, we can get $l_{1}(n) \leq \frac{\left(\alpha_{2}-\alpha\right) l_{3}(n) \log B}{\left(\alpha-\alpha_{1}\right) \log A}$, which yields

$$
\alpha_{1} l_{1}(n) \log A+\alpha_{2} l_{3}(n) \log B \geq \alpha\left(l_{1}(n) \log A+l_{3}(n) \log B\right) .
$$


For $n$ large enough, on the one hand,

$$
\begin{aligned}
& \frac{\sum_{k=1}^{n} x_{k}}{\sum_{k=1}^{n} b_{k}} \\
= & \frac{\alpha_{1} l_{1}(n) \log A-\frac{(\log q+\log (1-q)) l_{2}(n)}{2}+\alpha_{2} l_{3}(n) \log B}{l_{1}(n) \log A+\left(l_{2}(n)+l_{3}(n)\right) \log B} \\
\geq & \frac{\left(\alpha_{1} l_{1}(n) \log A+\alpha_{2} l_{3}(n) \log B\right)-\frac{(\log q+\log (1-q)) l_{2}(n)}{2}}{\left(l_{1}(n) \log A+l_{3}(n) \log B\right)+l_{2}(n) \log B},
\end{aligned}
$$

where the inequality from (4). Now we can get

$$
\frac{\sum_{k=1}^{n} x_{k}}{\sum_{k=1}^{n} b_{k}} \geq \alpha,
$$

since the inequlity in (1).

On the other hand, for $n=N_{2 i+1}^{\prime}$, we notice that

$$
l_{1}(n) \geq \frac{\left(\alpha_{2}-\alpha\right) l_{3}(n) \log B}{\left(\alpha-\alpha_{1}\right) \log A}-1,
$$

then

$$
\begin{aligned}
& \frac{\sum_{k=1}^{n} x_{k}}{\sum_{k=1}^{n} b_{k}} \\
= & \frac{\alpha_{1} l_{1}(n) \log A-\frac{(\log q+\log (1-q)) l_{2}(n)}{2}+\alpha_{2} l_{3}(n) \log B}{l_{1}(n) \log A+\left(l_{2}(n)+l_{3}(n)\right) \log B} \\
= & \frac{\left(\alpha_{1}\left(l_{1}(n)+1\right) \log A+\alpha_{2} l_{3}(n) \log B\right)-\alpha_{1} \log A-\frac{(\log q+\log (1-q)) l_{2}(n)}{2}}{\left(l_{1}(n) \log A+l_{3}(n) \log B\right)+l_{2}(n) \log B} \\
\leq & \frac{\alpha\left(\left(l_{1}(n)+1\right) \log A+l_{3}(n) \log B\right)-\alpha_{1} \log A-\frac{(\log q+\log (1-q)) l_{2}(n)}{2}}{\left(\left(l_{1}(n)+1\right) \log A+l_{3}(n) \log B\right)-\log A+l_{2}(n) \log B}
\end{aligned}
$$

The assumption $\lim _{i \rightarrow \infty} \frac{N_{i+1}}{N_{i}}=\infty$ yields $\lim _{i \rightarrow \infty} \frac{l_{2}\left(N_{2 i+1}^{\prime}\right) \log B}{l_{1}\left(N_{2 i+1}^{\prime}\right) \log A+l_{3}\left(N_{2 i+1}^{\prime}\right) \log B}=0$, and then limsup $\sup _{i \rightarrow \infty} \frac{\sum_{k=1}^{N_{2 i+1}^{\prime}} x_{k}}{\sum_{k=1}^{N_{2 i+1}^{\prime}} b_{k}} \leq \alpha$. Noticing inequality (5), we have

$$
\liminf _{n \rightarrow \infty} \frac{\sum_{k=1}^{n} x_{k}}{\sum_{k=1}^{n} b_{k}}=\alpha .
$$

Hence $\mu^{\prime}(\underline{E}(\mu, \alpha))=1$ by lemma 3.2 and 3.4 . A similar method yields

$$
\underline{\operatorname{dim}}_{\mathrm{loc}}\left(\mu^{\prime}, x\right)=\min \left\{\alpha, \frac{\log 2}{\log A}\right\}
$$

for $\mu^{\prime}$-almost every $x \in X$.

We now can conclude that $\operatorname{dim}_{H} \underline{E}(\mu, \alpha) \geq \min \left\{\alpha, \frac{\log 2}{\log A}\right\}$ from [8, item (a) in Proposition 2.3].

(3) We now turn to consider $\bar{E}(\mu, \alpha)$.

- Upper bound for $\operatorname{dim}_{H}(\bar{E}(\mu, \alpha))$ : The one obvious upper bound is $\operatorname{dim}_{H} X=$ $\frac{\log 2}{\log A}$ and the other is the following. 
- For $\alpha \in\left[\beta_{2}^{\prime}(1), \beta_{2}^{\prime}(-\infty)\right]$. noticing the result in [1, Proposition $1.3 \&$ inequality (1.5)], we know that

$$
\operatorname{dim}_{H} \bar{E}(\mu, \alpha) \leq \bar{f}_{\mu}^{L D}(\alpha, \alpha) \leq \underline{\tau}^{*}(\alpha)=\beta_{2}^{*}(\alpha) .
$$

- For $\alpha \in\left[\beta_{2}^{\prime}(+\infty), \beta_{2}^{\prime}(1)\right)$, the proof is the same as the proof of the upper bound for $\operatorname{dim}_{H}(\underline{E}(\mu, \alpha))$ with $\alpha \in\left[\beta_{1}^{\prime}(0), \beta_{1}^{\prime}(-\infty)\right]$, so we just list the key points.

Notice that $\operatorname{dim}_{H} \bar{E}(\mu, \alpha) \leq \underline{f}_{\mu}^{L D}(0, \alpha):=f_{2}(\alpha)$. Since $\beta_{2}^{*}(\alpha)=\inf _{s \geq 1}\{s \alpha-$ $\left.\beta_{2}(s)\right\}$, we just need to prove $\beta_{2}(s) \leq\left(f_{2}\right)^{*}(s)$ for $s \in[1,+\infty]$. If $\left\{B\left(x_{i}, r\right)\right\}$ is a packing of $\operatorname{supp}(\mu)$ by disjoint balls, we have for any $t \in \mathbb{R}$,

$$
\sum_{i} \mu\left(B\left(x_{i}, r\right)\right)^{s} \geq\left(\#\left\{i: \mu\left(B\left(x_{i}, r\right)\right) \geq r^{t+\epsilon}\right\}\right) r^{s(t+\epsilon)} .
$$

and $\beta_{2}(s)=\bar{\tau}(s)$ for $s \in[1,+\infty]$.

- The uppper bound for $\operatorname{dim}_{P}(\bar{E}(\mu, \alpha))$ :

Noticing [1, Proposition 1.3 \& inequality (1.5)], we have

$$
\operatorname{dim}_{P}(\bar{E}(\mu, \alpha)) \leq \sup \left\{\tau^{*}\left(\alpha^{\prime}\right): \alpha^{\prime} \leq \alpha\right\}= \begin{cases}\alpha & \alpha \in\left[\beta_{2}^{\prime}(+\infty), \beta_{2}^{\prime}(1)\right), \\ \beta_{2}^{*}(\alpha) & \alpha \in\left[\beta_{2}^{\prime}(1), \beta_{2}^{\prime}(0)\right) \\ \frac{\log 2}{\log B} & \alpha \in\left[\beta_{2}^{\prime}(0), \beta_{2}^{\prime}(-\infty)\right] .\end{cases}
$$

- Lower bound for $\operatorname{dim}_{H}(\bar{E}(\mu, \alpha))$ :

For $\alpha \in\left[\beta_{2}^{\prime}(+\infty), \beta_{2}^{\prime}(-\infty)\right]$, there exists $s \in \mathbb{R} \cup\{+\infty,-\infty\}$ such that $\beta_{2}^{\prime}(s)=$ $\alpha$, choose

$$
p_{n}^{\prime}=\left\{\begin{array}{ll}
1 / 2 & N_{2 i}<n \leq N_{2 i+1} \text { for some } i \in \mathbb{N} . \\
B^{\beta_{2}(s)} \cdot q^{s} & N_{2 i+1}<n \leq N_{2 i+2} \text { for some } i \in \mathbb{N}
\end{array} .\right.
$$

Now we have defined a measure $\mu^{\prime}$.

Noticing that

$$
\frac{-q^{\prime} \log q-\left(1-q^{\prime}\right) \log (1-q)}{\log B}=\beta_{2}^{\prime}(s)=\alpha,
$$

where $q^{\prime}=B^{\beta_{2}(s)} \cdot q^{s}$.

From lemma 3.4 and 3.2 and the choice of $s$, we get that $\mu^{\prime}(E(\mu, \alpha))=1$ and for $\mu^{\prime}$-almost every $x \in X$ one has $\underline{\operatorname{dim}}_{\mathrm{loc}}\left(\mu^{\prime}, x\right)=\min \left\{\frac{\log 2}{\log A}, \beta_{2}^{*}(\alpha)\right\}$. This gives

$$
\operatorname{dim}_{H} \underline{E}(\mu, \alpha) \geq \min \left\{\frac{\log 2}{\log A}, \beta_{2}^{*}(\alpha)\right\} .
$$

- Lower bound for $\operatorname{dim}_{P}(\bar{E}(\mu, \alpha))$ :

- For $\alpha \in\left[\beta_{2}^{\prime}(+\infty), \beta_{2}^{\prime}(1)\right)$, the proof is similar with the proof of the lower bound for $\operatorname{dim}_{H}(\underline{E}(\mu, \alpha))$ for $\alpha \in\left(\beta_{2}^{\prime}(1), \beta_{2}^{\prime}(0)\right)$, so we again just list the key points.

First, there exists $s \in(1,+\infty]$ such that $\beta_{2}^{\prime}(s)=\alpha$. 
Second, take $\alpha_{1}=\frac{-p \log p-(1-p) \log (1-p)}{\log A}$ and $\alpha_{2}=\frac{-q \log q-(1-q) \log (1-q)}{\log B}$. Define

$$
p_{n}^{\prime}= \begin{cases}p & N_{2 i}<n \leq N_{2 i+1} \text { for some } i \in \mathbb{N}, \\ q & N_{2 i+1}<n \leq N_{2 i+1}^{\prime} \text { for some } i \in \mathbb{N} \\ q^{s} B^{\beta_{2}(s)} & N_{2 i+1}^{\prime}<n \leq N_{2 i+2} \text { for some } i \in \mathbb{N}\end{cases}
$$

where $N_{2 i+1}^{\prime}=\min \left\{N_{2 i+2},\left\lfloor\frac{\left(\alpha_{2}-\alpha\right)\left(\sum_{k=0}^{i}\left(N_{2 k+1}-N_{2 k}\right)\right) \log A}{\left(\alpha-\alpha_{1}\right) \log B}\right\rfloor-\sum_{k=0}^{i-1}\left(N_{2 k+1}^{\prime}-\right.\right.$ $\left.\left.N_{2 k+1}\right)\right\}$. This also gives a measure $\mu^{\prime}$. We turn to analysis $\frac{\sum_{k=1}^{n} x_{k}}{\sum_{k=1}^{n} b_{k}}$. For any $n \in \mathbb{N}$, we assume that $l_{1}(n)=\#\left\{k \leq n: p_{k}^{\prime}=p\right\}, l_{2}(n) \stackrel{=}{=} \#\{k \leq$ $\left.n: p_{k}^{\prime}=q\right\}$ and $l_{3}(n)=\#\left\{k \leq n: p_{k}^{\prime}=q^{s} B^{\beta_{2}(s)}\right\}$ then $l_{1}(n)+l_{2}(n)+$ $l_{3}(n)=n$. In the same way, for $n$ large enough, we have

$$
\frac{\sum_{k=1}^{n} x_{k}}{\sum_{k=1}^{n} b_{k}} \leq \alpha
$$

and also we pay our attention to $n=N_{2 i+1}^{\prime}$

$$
\frac{\sum_{k=1}^{n} x_{k}}{\sum_{k=1}^{n} b_{k}} \geq \frac{\alpha\left(l_{1}(n) \log A+\left(l_{2}(n)+1\right) \log B\right)-\alpha_{2} \log B+\alpha l_{3}(n) \log B}{\left(l_{1}(n) \log A+\left(l_{2}(n)+1\right) \log B\right)-\log B+l_{3}(n) \log B} .
$$

Then, $\limsup _{i \rightarrow \infty} \frac{\sum_{k=1}^{N_{2 i}^{\prime}} x_{k}}{\sum_{k=1}^{N_{2 i}^{\prime} b_{k}}}=\alpha$. Hence $\mu^{\prime}(\bar{E}(\mu, \alpha))=1$ by lemma 3.2 and 3.4. A similar method yields $\overline{\operatorname{dim}}_{\mathrm{loc}}\left(\mu^{\prime}, x\right)=\max \left\{\alpha, \beta_{2}^{*}(\alpha)\right\}$ for $\mu^{\prime}$ almost every $x \in X$. We now get that $\operatorname{dim}_{P} \bar{E}(\mu, \alpha) \geq \max \left\{\alpha, \beta_{2}^{*}(\alpha)\right\}$.

$-\alpha \in\left(\beta_{2}^{\prime}(1), \beta_{2}^{\prime}(-\infty)\right]$. There exists $s \in(1,+\infty]$ such that $\beta_{2}^{\prime}(s)=\alpha$. Let $\beta_{2}^{*}\left(\alpha_{0}\right)=\max \left\{\beta_{2}^{*}\left(\alpha^{\prime}\right): \alpha^{\prime} \leq \alpha\right\}$, we can assume that $\alpha_{0}=\beta_{2}^{\prime}\left(s_{0}\right)$. Define

$$
p_{n}^{\prime}= \begin{cases}1 / 2 & N_{2 i-1}<n \leq N_{2 i} \text { for some } i \in \mathbb{N}, \\ B^{\beta_{2}\left(s_{0}\right)} p^{s_{0}} & N_{2 i}<n \leq N_{2 i}^{\prime} \text { for some } i \in \mathbb{N}, \\ B^{\beta_{2}(s)} p^{s} & N_{2 i}^{\prime}<n \leq N_{2 i+1} \text { for some } i \in \mathbb{N} .\end{cases}
$$

Where $\left\{N_{2 i}^{\prime}\right\}_{i \in \mathbb{N}}$ is a sequence of numbers such that $N_{2 i}<N_{2 i}^{\prime} \leq N_{2 i+1}$, $\lim _{i \rightarrow \infty} \frac{N_{2 i}^{\prime}}{N_{2 i}}=+\infty$ and $\lim _{i \rightarrow \infty} \frac{N_{2 i+1}}{N_{2 i}^{\prime}}=+\infty$. This can be done since $\lim _{i \rightarrow \infty} \frac{N_{i+1}}{N_{i}}=+\infty$. Now we can check that $\lim \sup _{n \rightarrow \infty} \frac{\sum_{k=1}^{n} x_{k}}{\sum_{k=1}^{n} b_{k}}=\alpha$ since $\lim _{i \rightarrow \infty} \frac{N_{2 i+1}}{N_{2 i}^{\prime}}=+\infty$ and $\alpha \geq \alpha_{0} \geq \frac{\log 2}{\log A}$. Hence $\mu^{\prime}(\bar{E}(\mu, \alpha))=1$ by lemma 3.2 and 3.4 Also, noticing that $\lim _{i \rightarrow \infty} \frac{N_{2 i}^{\prime}}{N_{2 i}}=+\infty$ and $\beta_{2}^{*}\left(\alpha_{0}\right)=$ $\max \left\{\beta_{2}^{*}\left(\alpha^{\prime}\right): \alpha^{\prime} \leq \alpha\right\} \geq \max \left\{\beta_{2}^{*}(\alpha), \frac{\log 2}{\log A}\right\}$, a similar method yields $\overline{\operatorname{dim}}_{\text {loc }}\left(\mu^{\prime}, x\right)=\beta_{2}^{*}\left(\alpha_{0}\right)$ for $\mu^{\prime}$-almost every $x \in X$. We now get that $\operatorname{dim}_{P} \bar{E}(\mu, \alpha) \geq \max \left\{\beta_{2}^{*}\left(\alpha^{\prime}\right): \alpha^{\prime} \leq \alpha\right\}$.

Remark 4.2. (1) In fact, the upper bound can also be obtained if we use the result in [11, Proposition $2.5 \& 2.6]$. (In our notation, the lower and upper $L^{q}$-spectrum plays the same roles as $-B$ and -b, see [2] for details, but we need to recall the functions $b, B$ and show their relationships with the functions $\bar{\tau}, \underline{\tau}$.) 
(2) For the Hausdorff dimension of the level sets $E(\mu, \alpha)$, the lower bound can be easily obtained by using the auxiliary measure $\mu^{\prime}$ as the classical method when $\alpha \in\left[\beta_{1}^{\prime}(+\infty), \beta_{1}^{\prime}(1)\right] \cup$ $\left[\beta_{1}^{\prime}(0), \beta_{1}^{\prime}(-\infty)\right]$. But it is a trouble for the part $\left.\left[\beta_{1}^{\prime}(1), \beta_{1}^{\prime}(0)\right]\right]$. This part is surprising to the author when it turns out to be $\min \left\{\alpha, \operatorname{dim}_{H} X\right\}$ which is related to two points $-\frac{q \log q+(1-q) \log (1-q)}{\log A}$ and $-\frac{p \log p+(1-p) \log (1-p)}{\log B}$. Until now the upper bound is easy for the part $\alpha \in\left[\beta_{1}^{\prime}(+\infty), \beta_{1}^{\prime}(0)\right]$, since $\bar{f}_{\mu}^{L D}(\alpha, \alpha)$ (also $\tau_{\mu}^{*}(\alpha)=\beta_{1}^{*}(\alpha)$ ) gives the sharp bound, but it is not easy for $\left[\beta_{1}^{\prime}(0), \beta_{1}^{\prime}(-\infty)\right]$. In this part the Legendre transform $\tau^{*}$ is not the sharp upper bound, so we turn to the lower large derivation spectrum $\underline{f}_{\mu}^{L D}(\alpha,+\infty)$ and prove that it coincides to $\bar{\tau}^{*}=\beta_{1}^{*}$ in this part.

For packing dimension, it is equal to that of the whole space, since we almost do not disturb the part when $N_{2 i+1}<n \leq N_{2 i+2}$ for $\underline{E}(\mu, \alpha)$.

(3) For the level sets $\bar{E}(\mu, \alpha)$, there are some dualities as for $\underline{E}(\mu, \alpha)$.

The Hausdorff dimension is from a traditional method except for the upper bound when $\alpha \in\left[\beta_{2}^{\prime}(+\infty), \beta_{2}^{\prime}(1)\right)$ where we borrow $\underline{f}_{\mu}^{L D}(0, \alpha)$.

The difficulty comes from the lower bound of $\operatorname{dim}_{P} \bar{E}(\mu, \alpha)$ for the part $\left[\beta_{2}^{\prime}(+\infty), \beta_{2}^{\prime}(1)\right]$, where we again deal with the linear part by the two points $-\frac{p \log p+(1-p) \log (1-p)}{\log A}$ and $-\frac{q \log q+(1-q) \log (1-q)}{\log B}$.

4.3. Proof of Theorem 2.5. The method to construct a corresponding auxiliary measure can get the sharp lower bound. A clear upper bound can be obtained if we notice that $E\left(\mu, \alpha, \alpha^{\prime}\right)=E(\mu, \alpha) \cap \bar{E}\left(\mu, \alpha^{\prime}\right)$. But it is not sharp for the Hausdorff dimension when $\left(\alpha, \alpha^{\prime}\right) \in\left[\beta_{1}^{\prime}(1), \beta_{1}^{\prime}(0)\right] \times\left[\beta_{2}^{\prime}(-\infty), \beta_{2}^{\prime}(1)\right)$ and for the packing dimension when $\left(\alpha, \alpha^{\prime}\right) \in$ $\left(\beta_{1}^{\prime}(1), \beta_{1}^{\prime}(-\infty)\right] \times\left[\beta_{2}^{\prime}(+\infty), \beta_{2}^{\prime}(1)\right)$. To obtain the upper bound in these situations we need to use [8, Proposition 2.3].

Proof. (1) Now we consider the Hausdorff dimension for $E\left(\mu, \alpha, \alpha^{\prime}\right)$.

- $\left(\alpha, \alpha^{\prime}\right) \notin\left[\beta_{1}^{\prime}(1), \beta_{1}^{\prime}(0)\right] \times\left[\beta_{2}^{\prime}(+\infty), \beta_{2}^{\prime}(1)\right)$

The upper bound can be obtained easily since $E\left(\mu, \alpha, \alpha^{\prime}\right)=\underline{E}(\mu, \alpha) \cap \bar{E}\left(\mu, \alpha^{\prime}\right)$. Let us focus our attention to the lower bound.

If $\alpha \notin\left[\beta_{1}^{\prime}(1), \beta_{1}^{\prime}(0)\right]$, the lower bound can be obtained by the following auxiliary measure $\mu^{\prime}$ defined thought $p_{n}^{\prime}$ with

$$
p_{n}^{\prime}= \begin{cases}A^{\beta_{1}\left(s_{1}\right)} p^{s_{1}} & N_{2 i}<n \leq N_{2 i+1} \text { for some } i \in \mathbb{N}, \\ B^{\beta_{2}\left(s_{2}\right)} q^{s_{2}} & N_{2 i+1}<n \leq N_{2 i+2} \text { for some } i \in \mathbb{N},\end{cases}
$$

where $\beta_{1}^{\prime}\left(s_{1}\right)=\alpha, \beta_{2}^{\prime}\left(s_{2}\right)=\alpha^{\prime}$. It is easy to show that $\mu^{\prime}\left(E\left(\mu, \alpha, \alpha^{\prime}\right)\right)=1$ and $\operatorname{dim}_{H} \mu^{\prime} \geq \min \left\{\beta_{1}^{*}(\alpha), \beta_{2}^{*}\left(\alpha^{\prime}\right)\right\}=\min \left\{\operatorname{dim}_{H}(\underline{E}(\mu, \alpha)), \operatorname{dim}_{H}\left(\bar{E}\left(\mu, \alpha^{\prime}\right)\right)\right\}$. The result yields since $\operatorname{dim}_{H} E\left(\mu, \alpha, \alpha^{\prime}\right) \geq \operatorname{dim}_{H} \mu^{\prime}$.

If $\alpha \in\left[\beta_{1}^{\prime}(1), \beta_{1}^{\prime}(0)\right]$ and $\alpha^{\prime} \notin\left[\beta_{2}^{\prime}(+\infty), \beta_{2}^{\prime}(1)\right)$, choose $s_{2} \in[-\infty, 1]$ such that $\beta_{2}^{\prime}\left(s_{2}\right)=\alpha^{\prime}$, let

$$
p_{n}^{\prime}= \begin{cases}p & N_{2 i}<n \leq N_{2 i}^{\prime} \text { for some } i \in \mathbb{N}, \\ 1 / 2 & N_{2 i}^{\prime}<n \leq N_{2 i+1} \text { for some } i \in \mathbb{N}, \\ B^{\beta_{2}\left(s_{2}\right)} q^{s_{2}} & N_{2 i+1}<n \leq N_{2 i+1}^{\prime} \text { for some } i \in \mathbb{N}, \\ q & N_{2 i+1}^{\prime}<n \leq N_{2 i+2} \text { for some } i \in \mathbb{N},\end{cases}
$$


as a similar way in the lower bound for $\operatorname{dim}_{H} \underline{E}(\mu, \alpha)$ (Also need to choose proper $\left\{N_{i}^{\prime}\right\}_{i \in \mathbb{N}}$, but it is almost the same as in the proof of theorem 2.3, so we omit the details). Then we can obtain the lower bound.

- If $\alpha^{\prime} \in\left[\beta_{2}^{\prime}(+\infty), \beta_{2}^{\prime}(1)\right)$ with $g\left(\alpha^{\prime}\right) \leq \beta_{1}^{\prime}(0)$ and $\alpha \in\left[g\left(\alpha^{\prime}\right), \beta_{1}^{\prime}(0)\right)$. Recall that we take the tangent to the graph $\beta_{1}^{*}$ passing through the point $\left(\alpha^{\prime}, \beta_{2}^{*}\left(\alpha^{\prime}\right)\right)$ and denote the point of tangency by $\left(g\left(\alpha^{\prime}\right), \beta_{1}^{*}\left(g\left(\alpha^{\prime}\right)\right)\right)=:\left(\alpha_{1}, \beta_{1}^{*}\left(\alpha_{1}\right)\right)$. Then take $s_{1} \in[0,1], s_{2} \in(1,+\infty)$ such that $\beta_{1}^{\prime}\left(s_{1}\right)=\alpha_{1}$ and $\beta_{2}^{\prime}\left(s_{2}\right)=\alpha^{\prime}$.

Choose proper $\left\{N_{2 i}^{\prime}\right\}_{i \in \mathbb{N}}$, let

$$
p_{n}^{\prime}= \begin{cases}A^{\beta_{1}\left(s_{1}\right)} p^{s_{1}} & N_{2 i}<n \leq N_{2 i}^{\prime} \text { for some } i \in \mathbb{N}, \\ 1 / 2 & N_{2 i}^{\prime}<n \leq N_{2 i+1} \text { for some } i \in \mathbb{N}, \\ B^{\beta_{2}\left(s_{2}\right)} q^{s_{2}} & N_{2 i+1}<n \leq N_{2 i+2} \text { for some } i \in \mathbb{N},\end{cases}
$$

We can obtain that $\operatorname{dim}_{H} E\left(\mu, \alpha, \alpha^{\prime}\right) \geq \min \left\{\frac{\log 2}{\log A}, s_{1} \alpha-\beta_{1}\left(s_{1}\right)\right\}$ in the same way as in the proof of theorem 2.3 .

Now we turn to the upper bound, a crucial observation is $\left(\beta_{i}^{*}\right)^{\prime}\left(\beta_{i}^{\prime}(s)\right)=s$ for any $s \in \mathbb{R}$ and $i=1,2$. It is easily obtained by differentiation of a composition function. Let

$$
p_{n}^{\prime}= \begin{cases}A^{\beta_{1}\left(s_{1}\right)} p^{s_{1}} & N_{2 i}<n \leq N_{2 i+1} \text { for some } i \in \mathbb{N}, \\ B^{\beta_{2}\left(s_{2}\right)} q^{s_{2}} & N_{2 i+1}<n \leq N_{2 i+2} \text { for some } i \in \mathbb{N} .\end{cases}
$$

This defines a measure denoted by $\mu^{\prime}$. Now we want to show that for any $x \in E\left(\mu, \alpha, \alpha^{\prime}\right)$ we have $\underline{\operatorname{dim}}_{\text {loc }}\left(\mu^{\prime}, x\right) \leq s_{1} \alpha-\beta_{1}\left(s_{1}\right)$. If so, the upper bound is obtained from [8, Proposition $2.3(\mathrm{~b})]$ and $\operatorname{dim}_{H} X=\frac{\log 2}{\log A}$.

Fix $x \in E\left(\mu, \alpha, \alpha^{\prime}\right)$ that is for any $\epsilon>0$, there exists $N \in \mathbb{N}$ such that for any $n \geq N, d(\mu, x, n) \leq \alpha^{\prime}+\epsilon$, also for any $N^{\prime} \in \mathbb{N}$, there exists $n^{\prime} \geq N^{\prime}$ with $d\left(\mu, x, n^{\prime}\right) \leq \alpha+\epsilon$.

Choose $i_{0}$ large enough with $N_{2 i_{0}}>N$ such that for any $i \geq i_{0}$ one has

$$
\begin{gathered}
1 \leq \frac{\log \mu\left(I_{N_{2 i}}(x)\right)}{\log \mu\left(I_{N_{2 i}}(x)\right)-\log \mu\left(I_{N_{2 i-1}}(x)\right)} \leq 1+\epsilon \\
1 \leq \frac{\log \mu^{\prime}\left(I_{N_{2 i}}(x)\right)}{\log \mu^{\prime}\left(I_{N_{2 i}}(x)\right)-\log \mu^{\prime}\left(I_{N_{2 i-1}}(x)\right)} \leq 1+\epsilon \\
1 \leq \frac{\log \left|I_{N_{2 i}}(x)\right|}{\log \left|I_{N_{2 i}}(x)\right|-\log \left|I_{N_{2 i-1}}(x)\right|} \leq 1+\epsilon
\end{gathered}
$$

For any $n>N_{2 i_{0}}$ such that $d(\mu, x, n) \leq \alpha+\epsilon$, choose the largest $i$ with $N_{2 i} \leq$ $n$, one has $\alpha_{2}:=d\left(\mu, x, N_{2 i}\right) \leq \alpha^{\prime}+\epsilon$. Now we assume that

$$
\frac{\log \mu\left(I_{n}(x)\right)-\log \mu\left(I_{N_{2 i}}(x)\right)}{\log \left|I_{n}(x)\right|-\log \left|I_{N_{2 i}}(x)\right|}=: \alpha_{0} .
$$

Then we have:

$$
\frac{\alpha_{2} \log \left(\left|I_{N_{2 i}}(x)\right|\right)+\alpha_{0}\left(\log \left|I_{n}(x)\right|-\log \left|I_{N_{2 i}}(x)\right|\right)}{\log \left(\left|I_{N_{2 i}}(x)\right|\right)+\left(\log \left|I_{n}(x)\right|-\log \left|I_{N_{2 i}}(x)\right|\right)} \leq \alpha+\epsilon .
$$

Let us illustrate these relationships by figure 8 


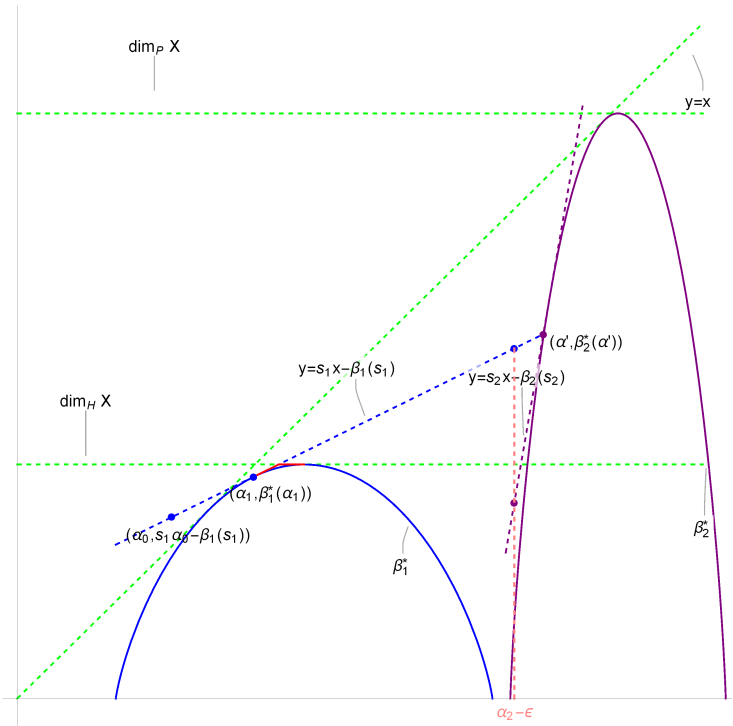

FIGURE 8.

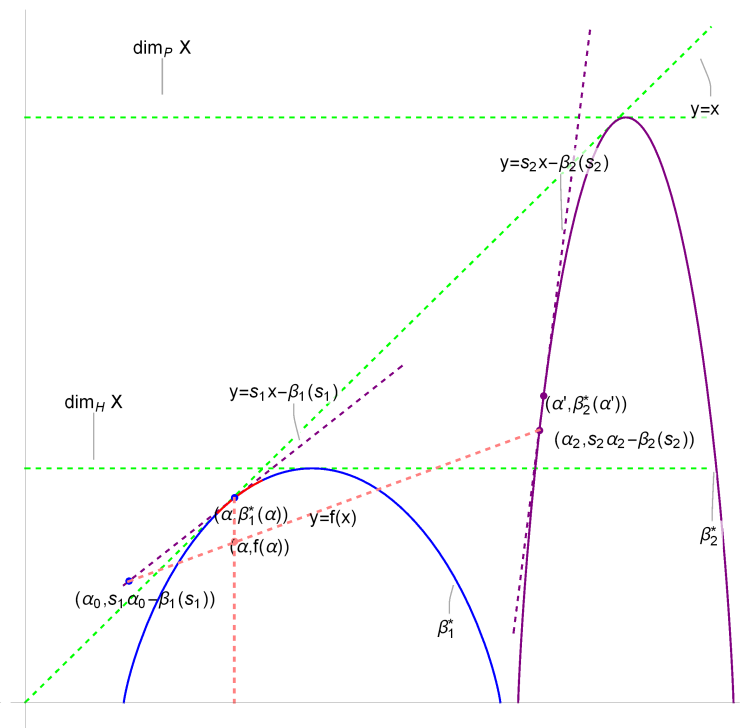

FIGURE 9.

Now we turn to estimate $\log \mu^{\prime}\left(I_{N_{2 i}}(x)\right)$. First we notice inequality (7) and the definition of $\mu^{\prime}$, one has

$$
\begin{aligned}
\log \mu^{\prime}\left(I_{N_{2 i}}(x)\right) \geq & \left(\log \mu^{\prime}\left(I_{N_{2 i}}(x)\right)-\log \mu^{\prime}\left(I_{N_{2 i-1}}(x)\right)\right)(1+\epsilon) \\
= & \left(s_{2}\left(\log \mu\left(I_{N_{2 i}}(x)\right)-\log \mu\left(I_{N_{2 i-1}}(x)\right)\right)\right)(1+\epsilon) \\
& -\beta_{2}\left(s_{2}\right)\left(\log \left|I_{N_{2 i}}(x)\right|-\log \left|I_{N_{2 i-1}}(x)\right|\right)(1+\epsilon) .
\end{aligned}
$$

Second, since $s_{2}>1, \beta_{2}\left(s_{2}\right)>0$ and inequality (6) and (8), one has

$$
\begin{aligned}
\log \mu^{\prime}\left(I_{N_{2 i}}(x)\right) & \geq s_{2}(1+\epsilon) \log \mu\left(I_{N_{2 i}}(x)\right)-(1+\epsilon) \beta_{2}\left(s_{2}\right) \log \left|I_{N_{2 i}}(x)\right| \\
& =s_{2} \alpha_{2}(1+\epsilon) \log \left|I_{N_{2 i}}(x)\right|-(1+\epsilon) \beta_{2}\left(s_{2}\right) \log \left|I_{N_{2 i}}(x)\right| \\
& =\left(s_{2} \alpha_{2}-\beta_{2}\left(s_{2}\right)\right)(1+\epsilon) \log \left|I_{N_{2 i}}(x)\right|
\end{aligned}
$$

Third, we notice $\alpha_{2} \leq \alpha^{\prime}+\epsilon$, i.e. $\alpha_{2}-\epsilon \leq \alpha^{\prime}$, then from figure 10 , we have

$$
\left.s_{2}\left(\alpha_{2}-\epsilon\right)-\beta_{2}\left(s_{2}\right) \leq s_{1}\left(\alpha_{2}-\epsilon\right)\right)-\beta_{1}\left(s_{1}\right) \leq s_{1} \alpha_{2}-\beta_{1}\left(s_{1}\right) .
$$

That is $s_{2} \alpha_{2}-\beta_{2}\left(s_{2}\right) \leq s_{1} \alpha_{2}-\beta_{1}\left(s_{1}\right)+S_{2} \epsilon$. So

$$
\log \mu^{\prime}\left(I_{N_{2 i}}(x)\right) \geq\left(s_{1} \alpha_{2}-\beta_{1}\left(s_{1}\right)+s_{2} \epsilon\right)(1+\epsilon) \log \left|I_{N_{2 i}}(x)\right| .
$$

Also from the construction of $\mu^{\prime}$ one has

$$
\log \mu^{\prime}\left(I_{n}(x)\right)-\log \mu^{\prime}\left(I_{N_{2 i}}(x)\right)=\left(s_{1} \alpha_{0}-\beta_{1}\left(s_{1}\right)\right)\left(\log \left|I_{n}(x)\right|-\log \left|I_{N_{2 i}}(x)\right|\right)
$$


Then

$$
\begin{aligned}
& d\left(\mu^{\prime}, x, n\right) \\
= & \frac{\log \mu^{\prime}\left(I_{n}(x)\right)}{\log \left(\left|I_{n}(x)\right|\right)} \\
= & \frac{\log \mu^{\prime}\left(I_{N_{2 i}}(x)\right)+\log \mu^{\prime}\left(I_{n}(x)\right)-\log \mu^{\prime}\left(I_{N_{2 i}}(x)\right)}{\log \left(\left|I_{N_{2 i}}(x)\right|\right)+\left(\log \left|I_{n}(x)\right|-\log \left|I_{N_{2 i}}(x)\right|\right)} \\
\leq & \frac{\left(s_{1} \alpha_{2}-\beta_{1}\left(s_{1}\right)+s_{2} \epsilon\right)(1+\epsilon) \log \left|I_{N_{2 i}}(x)\right|+\left(s_{1} \alpha_{0}-\beta_{1}\left(s_{1}\right)\right)\left(\log \left|I_{n}(x)\right|-\log \left|I_{N_{2 i}}(x)\right|\right)}{\log \left(\left|I_{N_{2 i}}(x)\right|\right)+\left(\log \left|I_{n}(x)\right|-\log \left|I_{N_{2 i}}(x)\right|\right)}
\end{aligned}
$$

(here we use inequality (10) and equation 11)

$\leq \frac{\left(s_{1} \alpha_{2}-\beta_{1}\left(s_{1}\right)\right) \log \left|I_{N_{2 i}}(x)\right|+\left(s_{1} \alpha_{0}-\beta_{1}\left(s_{1}\right)\right)\left(\log \left|I_{n}(x)\right|-\log \left|I_{N_{2 i}}(x)\right|\right)}{\log \left(\left|I_{N_{2 i}}(x)\right|\right)+\left(\log \left|I_{n}(x)\right|-\log \left|I_{N_{2 i}}(x)\right|\right)}$

$+s_{2} \epsilon(1+\epsilon)+\left(s_{1} \alpha_{2}-\beta_{1}\left(s_{1}\right)\right) \epsilon$

$\leq \frac{s_{1} \alpha_{2} \log \left|I_{N_{2 i}}(x)\right|+s_{1} \alpha_{0}\left(\log \left|I_{n}(x)\right|-\log \left|I_{N_{2 i}}(x)\right|\right)}{\log \left(\left|I_{N_{2 i}}(x)\right|\right)+\left(\log \left|I_{n}(x)\right|-\log \left|I_{N_{2 i}}(x)\right|\right)}-\beta_{1}\left(s_{1}\right)$

$+s_{2} \epsilon(1+\epsilon)+\left(s_{1} \alpha_{2}-\beta_{1}\left(s_{1}\right)\right) \epsilon$

$\leq s_{1}(\alpha+\epsilon)-\beta_{1}\left(s_{1}\right)+s_{2} \epsilon(1+\epsilon)+\left(s_{1} \alpha_{2}-\beta_{1}\left(s_{1}\right)\right) \epsilon$

(see inequality (9))

That is

$$
d\left(\mu^{\prime}, x, n\right) \leq s_{1}(\alpha+\epsilon)-\beta_{1}\left(s_{1}\right)+s_{2} \epsilon(1+\epsilon)+\left(s_{1} \alpha_{2}-\beta_{1}\left(s_{1}\right)\right) \epsilon
$$

for any $n>N_{2 i_{0}}$ with $d(\mu, x, n) \leq \alpha+\epsilon$.

Noticing for any $N^{\prime} \in \mathbb{N}$, there exists $n \geq N^{\prime}$ with $d(\mu, x, n) \leq \alpha+\epsilon$, so that

$$
\liminf _{n \rightarrow \infty} d\left(\mu^{\prime}, x, n\right) \leq s_{1} \alpha-\beta_{1}\left(s_{1}\right),
$$

and then $\operatorname{dim}_{\mathrm{loc}}\left(\mu^{\prime}, x\right) \leq s_{1} \alpha-\beta_{1}\left(s_{1}\right)$ from lemma 3.4 .

- $\alpha^{\prime} \in\left[\beta_{2}^{\prime}(+\infty), \beta_{2}^{\prime}(1)\right)$ and $\alpha \in\left[\beta_{1}^{\prime}(1), \min \left\{g\left(\alpha^{\prime}\right), \frac{\log 2}{\log A}\right\}\right)$. The lower bound can be obtained by the following auxiliary measure $\mu^{\prime}$ defined thought $p_{n}^{\prime}$ with

$$
p_{n}^{\prime}= \begin{cases}A^{\beta_{1}\left(s_{1}\right)} p^{s_{1}} & N_{2 i}<n \leq N_{2 i+1} \text { for some } i \in \mathbb{N}, \\ B^{\beta_{2}\left(s_{2}\right)} q^{s_{2}} & N_{2 i+1}<n \leq N_{2 i+2} \text { for some } i \in \mathbb{N},\end{cases}
$$

For the lower bound, Use the same method we can also define the point $\left(\alpha_{0}, s_{1} \alpha_{0}-\beta_{1}\left(s_{1}\right)\right)$ and $\left(\alpha_{2}, s_{2} \alpha_{2}-\beta_{2}\left(s_{2}\right)\right)$. We now replace the tangent in the previous item by the line passing through these two points. We assume the line is the graph of the linear function $y=f(x)$. Let us illustrate these relationships by figure 9 .

The proof is almost the same as before to get that for any $x \in E\left(\mu, \alpha, \alpha^{\prime}\right)$ we have $\underline{\operatorname{dim}}_{\text {loc }}\left(\mu^{\prime}, x\right) \leq f(\alpha)$, but we also need to use the inequality

$$
f(\alpha) \leq \beta_{1}^{*}(\alpha) .
$$

Also $\beta_{2}^{*}\left(\alpha^{\prime}\right)$ is a travail bound since $E\left(\mu, \alpha, \alpha^{\prime}\right) \subset \bar{E}\left(\mu, \alpha^{\prime}\right)$. So that the upper bound can be obtained.

(2) Now we turn to $\operatorname{dim}_{P} E\left(\mu, \alpha, \alpha^{\prime}\right)$. We assume that $\beta_{1}^{\prime}\left(s_{1}\right)=\alpha, \beta_{2}^{\prime}\left(s_{2}\right)=\alpha^{\prime}$ 
- $\left(\alpha, \alpha^{\prime}\right) \notin\left(\beta_{1}^{\prime}(1), \beta_{1}^{\prime}(-\infty)\right] \times\left[\beta_{2}^{\prime}(+\infty), \beta_{2}^{\prime}(1)\right)$ :

If $\alpha^{\prime} \notin\left[\beta_{2}^{\prime}(+\infty), \beta_{2}^{\prime}(1)\right)$, let $\beta_{2}^{*}\left(\alpha_{0}\right)=\max \left\{\beta_{2}^{*}\left(\alpha_{1}\right): \alpha_{1} \leq \alpha^{\prime}\right\}$, we can assume that $\alpha_{0}=\beta_{2}^{\prime}\left(s_{0}\right)$. Using the same way as the proof of theorem 2.3, define

$$
p_{n}^{\prime}= \begin{cases}q^{s_{1}} A^{\beta_{1}\left(s_{1}\right)} & N_{2 i-1}<n \leq N_{2 i} \text { for some } i \in \mathbb{N}, \\ B^{\beta_{2}\left(s_{0}\right)} p^{s_{0}} & N_{2 i}<n \leq N_{2 i}^{\prime} \text { for some } i \in \mathbb{N}, \\ B^{\beta_{2}(s)} p^{s} & N_{2 i}^{\prime}<n \leq N_{2 i+1} \text { for some } i \in \mathbb{N} .\end{cases}
$$

Where $\left\{N_{2 i}^{\prime}\right\}_{i \in \mathbb{N}}$ is a sequence of numbers such that $N_{2 i}<N_{2 i}^{\prime} \leq N_{2 i+1}$, $\lim _{i \rightarrow \infty} \frac{N_{2 i}^{\prime}}{N_{2 i}}=+\infty$ and $\lim _{i \rightarrow \infty} \frac{N_{2 i+1}}{N_{2 i}^{\prime}}=+\infty$. This can be done since $\lim _{i \rightarrow \infty} \frac{N_{i+1}}{N_{i}}=$ $+\infty$. We now define a measure $\mu^{\prime}$ and can prove that $\mu^{\prime}\left(E\left(\mu, \alpha, \alpha^{\prime}\right)\right)=1$ and for $\mu^{\prime}$-a.e. $x \in X, \overline{\operatorname{dim}}_{\text {loc }}\left(\mu^{\prime}, x\right) \geq \beta_{2}^{*}\left(\alpha_{0}\right)$. Then, from [8, Proposition 2.3], we have $\operatorname{dim}_{P} E\left(\mu, \alpha, \alpha^{\prime}\right) \geq \beta_{2}^{*}\left(\alpha_{0}\right)$. Since $E\left(\mu, \alpha, \alpha^{\prime}\right) \subset \bar{E}\left(\alpha^{\prime}\right)$ and $\operatorname{dim}_{P} \bar{E}\left(\mu, \alpha^{\prime}\right)=\beta_{2}^{*}\left(\alpha_{0}\right)$, we get that

$$
\operatorname{dim}_{P} E\left(\mu, \alpha, \alpha^{\prime}\right)=\operatorname{dim}_{P} \bar{E}\left(\mu, \alpha^{\prime}\right) .
$$

If $\alpha^{\prime} \in\left[\beta_{2}^{\prime}(+\infty), \beta_{2}^{\prime}(1)\right)$ and $\alpha \notin\left(\beta_{1}^{\prime}(1), \beta_{1}^{\prime}(-\infty)\right]$, define

$$
p_{n}^{\prime}= \begin{cases}p^{s_{1}} A^{\beta_{1}\left(s_{1}\right)} & N_{2 i}<n \leq N_{2 i}^{\prime} \text { for some } i \in \mathbb{N} \\ p & N_{2 i}^{\prime}<n \leq N_{2 i+1} \text { for some } i \in \mathbb{N}, \\ q & N_{2 i+1}<n \leq N_{2 i+1}^{\prime} \text { for some } i \in \mathbb{N}, \\ q^{s_{2}} B^{\beta_{2}\left(s_{2}\right)} & N_{2 i+1}^{\prime}<n \leq N_{2 i+2} \text { for some } i \in \mathbb{N} .\end{cases}
$$

Where $\left\{N_{i}^{\prime}\right\}_{i \in \mathbb{N}}$ is a sequence of numbers such that $N_{i}<N_{i}^{\prime} \leq N_{i+1}, \lim _{i \rightarrow \infty} \frac{N_{i}^{\prime}}{N_{i}}=$ $+\infty$ and $\lim _{i \rightarrow \infty} \frac{N_{i+1}}{N_{i}^{\prime}}=+\infty$. This can be done since $\lim _{i \rightarrow \infty} \frac{N_{i+1}}{N_{i}}=+\infty$.

We now define a measure $\mu^{\prime}$, a similar method for the lower bound for $\operatorname{dim}_{P} \bar{E}\left(\mu, \alpha^{\prime}\right)$ and $E\left(\mu, \alpha, \alpha^{\prime}\right) \subset \bar{E}\left(\mu, \alpha^{\prime}\right)$ yield $\operatorname{dim}_{P} E\left(\mu, \alpha, \alpha^{\prime}\right)=\operatorname{dim}_{P} \bar{E}\left(\mu, \alpha^{\prime}\right)$.

- For $\alpha \in\left(\beta_{1}^{\prime}(1), \beta_{1}^{\prime}(-\infty)\right]$ and $\alpha^{\prime} \in\left[\beta_{2}^{\prime}(+\infty), h(\alpha)\right)$. Recall that we take the tangent to the graph $\beta_{2}^{*}$ passing through the point $\left(\alpha, \beta_{1}^{*}(\alpha)\right)$ and denote the point of tangency by $\left(h(\alpha), \beta_{2}^{*}(h(\alpha))\right)$. Then take $s_{2} \in(1,+\infty]$ such that $\beta_{2}^{\prime}\left(s_{2}\right)=h(\alpha)=: \alpha_{2}$ and $s_{1} \in[-\infty, 1)$ such that $\beta_{1}^{\prime}\left(s_{1}\right)=\alpha \cdot \operatorname{dim}_{P} E\left(\mu, \alpha, \alpha^{\prime}\right) \geq$ $s_{2} \alpha^{\prime}-\beta_{2}\left(s_{2}\right)$ can be obtained by using the following auxiliary measure $\mu^{\prime}$ though $\left\{p_{n}^{\prime}\right\}_{n \in \mathbb{N}}$ as the lower bound for $\operatorname{dim}_{P} \bar{E}\left(\mu, \alpha^{\prime}\right)$.

$$
p_{n}^{\prime}= \begin{cases}p^{s_{1}} A^{\beta_{1}\left(s_{1}\right)} & N_{2 i}<n \leq N_{2 i+1} \text { for some } i \in \mathbb{N} \\ q & N_{2 i+1}<n \leq N_{2 i+1}^{\prime} \text { for some } i \in \mathbb{N}, \\ q^{s_{2}} B^{\beta_{2}\left(s_{2}\right)} & N_{2 i+1}^{\prime}<n \leq N_{2 i+2} \text { for some } i \in \mathbb{N} .\end{cases}
$$

The upper bound is similar with the Hausdorff dimension in the second item, but noticing figure 10 . We need to show that for any $x \in E\left(\mu, \alpha, \alpha^{\prime}\right)$ we have $\overline{\operatorname{dim}}_{\text {loc }}\left(\mu^{\prime}, x\right) \leq s_{2} \alpha^{\prime}-\beta_{2}\left(s_{2}\right)$.

Fix $x \in E\left(\mu, \alpha, \alpha^{\prime}\right)$, that is for any $\epsilon>0$, there exists $N \in \mathbb{N}$ such that for any $n \geq N, d(\mu, x, n) \geq \alpha-\epsilon$ and $d(\mu, x, n) \leq \alpha^{\prime}+\epsilon$.

Choose $i_{0}$ with $N_{2 i_{0}+1}>N$ large enough such that for any $i \geq i_{0}$ one has

$$
1 \leq \frac{\log \mu\left(I_{N_{2 i+1}}(x)\right)}{\log \mu\left(I_{N_{2 i+1}}(x)\right)-\log \mu\left(I_{N_{2 i}}(x)\right)} \leq 1+\epsilon
$$




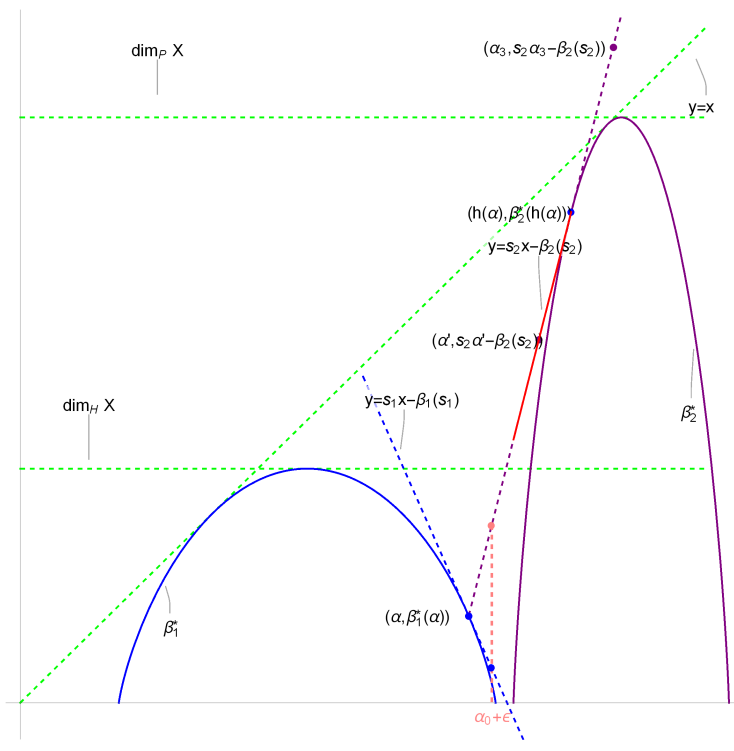

FIGURE 10.

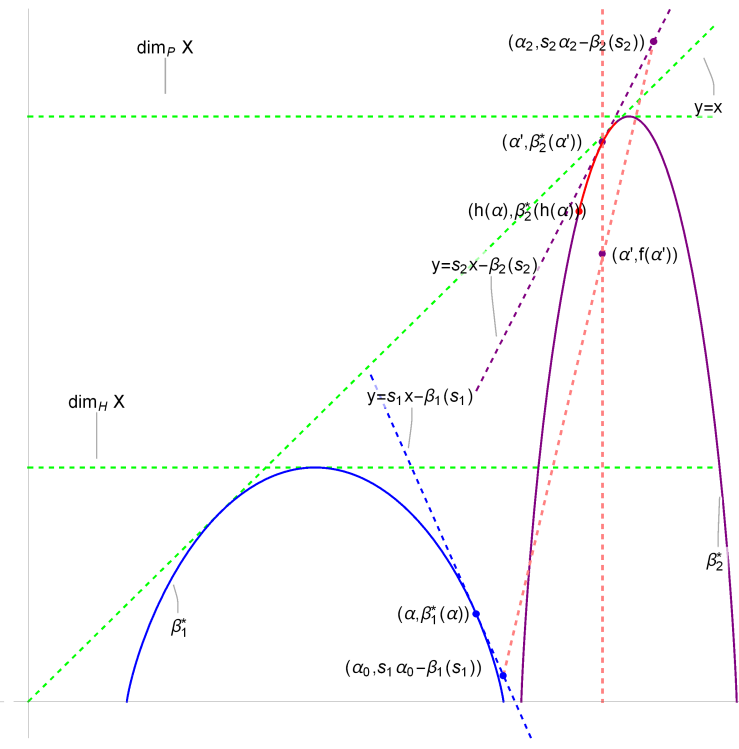

FIGURE 11.

$$
\begin{gathered}
1 \leq \frac{\log \mu^{\prime}\left(I_{N_{2 i+1}}(x)\right)}{\log \mu^{\prime}\left(I_{N_{2 i+1}}(x)\right)-\log \mu^{\prime}\left(I_{N_{2 i}}(x)\right)} \leq 1+\epsilon \\
1 \leq \frac{\log \left|I_{N_{2 i+1}}(x)\right|}{\log \left|I_{N_{2 i+1}}(x)\right|-\log \left|I_{N_{2 i}}(x)\right|} \leq 1+\epsilon
\end{gathered}
$$

For any $n>N_{2 i_{0}+1}$, one has $d(\mu, x, n) \leq \alpha^{\prime}+\epsilon$, choose the largest $i$ with $N_{2 i+1} \leq n$, one has $\alpha_{0}:=d\left(\mu, x, N_{2 i+1}\right) \geq \alpha-\epsilon$. Now we assume that

$$
\frac{\log \mu\left(I_{n}(x)\right)-\log \mu\left(I_{N_{2 i+1}}(x)\right)}{\log \left|I_{n}(x)\right|-\log \left|I_{N_{2 i+1}}(x)\right|}=: \alpha_{3} .
$$

Then we have:

$$
\frac{\alpha_{3} \log \left(\left|I_{N_{2 i+1}}(x)\right|\right)+\alpha_{0}\left(\log \left|I_{n}(x)\right|-\log \left|I_{N_{2 i+1}}(x)\right|\right)}{\log \left(\left|I_{N_{2 i+1}}(x)\right|\right)+\left(\log \left|I_{n}(x)\right|-\log \left|I_{N_{2 i+1}}(x)\right|\right)} \leq \alpha^{\prime}+\epsilon .
$$

Now we turn to estimate $\log \mu^{\prime}\left(I_{N_{2 i+1}}(x)\right)$.

First, we notice inequality (14) and the definition of $\mu^{\prime}$, one has

$$
\begin{aligned}
\log \mu^{\prime}\left(I_{N_{2 i+1}}(x)\right) \geq & \left(\log \mu^{\prime}\left(I_{N_{2 i+1}}(x)\right)-\log \mu^{\prime}\left(I_{N_{2 i}}(x)\right)\right)(1+\epsilon) \\
= & \left(s_{1}\left(\log \mu\left(I_{N_{2 i+1}}(x)\right)-\log \mu\left(I_{N_{2 i}}(x)\right)\right)\right)(1+\epsilon) \\
& -\beta_{1}\left(s_{1}\right)\left(\log \left|I_{N_{2 i+1}}(x)\right|-\log \left|I_{N_{2 i}}(x)\right|\right)(1+\epsilon) .
\end{aligned}
$$

Second, from inequality (13) and (15), one has

$$
\begin{aligned}
\log \mu^{\prime}\left(I_{N_{2 i+1}}(x)\right) & \geq\left(s_{1}+\left|s_{1}\right| \epsilon\right) \log \mu\left(I_{N_{2 i+1}}(x)\right)-(1+\epsilon) \beta_{1}\left(s_{1}\right) \log \left|I_{N_{2 i+1}}(x)\right| \\
& =\left(s_{1}+\left|s_{1}\right| \epsilon\right) \alpha_{0} \log \left|I_{N_{2 i}}(x)\right|-(1+\epsilon) \beta_{1}\left(s_{1}\right) \log \left|I_{N_{2 i+1}}(x)\right| \\
& =\left(\left(s_{1} \alpha_{0}-\beta_{1}\left(s_{1}\right)\right)+\left|s_{1}\right| \epsilon \alpha_{0}-\beta_{1}\left(s_{1}\right) \epsilon\right) \log \left|I_{N_{2 i}}(x)\right|
\end{aligned}
$$

Third, we notice $\alpha_{0} \geq \alpha-\epsilon$, i.e. $\alpha_{0}+\epsilon \geq \alpha$, then from figure 10, we have $\left.s_{1}\left(\alpha_{0}+\epsilon\right)-\beta_{1}\left(s_{1}\right) \leq s_{2}\left(\alpha_{0}+\epsilon\right)\right)-\beta_{2}\left(s_{2}\right) \leq s_{2} \alpha_{0}-\beta_{2}\left(s_{2}\right)+s_{2} \epsilon$. 
That is $s_{1} \alpha_{0}-\beta_{1}\left(s_{1}\right) \leq s_{2} \alpha_{0}-\beta_{2}\left(s_{2}\right)+s_{2} \epsilon+\left|s_{1}\right| \epsilon$. So

$\log \mu^{\prime}\left(I_{N_{2 i+1}}(x)\right) \geq\left(s_{2} \alpha_{0}-\beta_{2}\left(s_{2}\right)+\left(s_{2}+\left|s_{1}\right|\left(1+\alpha_{0}\right)+\beta_{1}\left(s_{1}\right)\right) \epsilon\right) \log \left|I_{N_{2 i+1}}(x)\right|$.

Also from the construction of $\mu^{\prime}$ and the definition of $\alpha_{3}$, one has

$$
\log \mu^{\prime}\left(I_{n}(x)\right)-\log \mu^{\prime}\left(I_{N_{2 i+1}}(x)\right)=\left(s_{2} \alpha_{3}-\beta_{2}\left(s_{2}\right)\right)\left(\log \left|I_{n}(x)\right|-\log \left|I_{N_{2 i+1}}(x)\right|\right)
$$

Then, using a similar method in the proof of inequality (12), but replace (10), (11) and (9) by (17), 18and (16), we can conclude

$$
d\left(\mu^{\prime}, x, n\right) \leq s_{2}\left(\alpha^{\prime}+\epsilon\right)-\beta_{2}\left(s_{2}\right)+s_{2} \epsilon(1+\epsilon)+\left(s_{1} \alpha_{3}-\beta_{1}\left(s_{1}\right)\right) \epsilon
$$

for any $n \geq N_{2 i_{0}+1}$. This gives

$$
\limsup _{n \rightarrow \infty} d\left(\mu^{\prime}, x, n\right) \leq s_{2} \alpha^{\prime}-\beta_{2}\left(s_{2}\right),
$$

and then $\overline{\operatorname{dim}}_{\mathrm{loc}}\left(\mu^{\prime}, x\right) \leq s_{2} \alpha^{\prime}-\beta_{2}\left(s_{2}\right)$ from lemma 3.4

- For $\alpha \in\left(\beta_{1}^{\prime}(1), \beta_{1}^{\prime}(-\infty)\right]$ and $\alpha^{\prime} \in\left[h(\alpha), \beta_{2}^{\prime}(1)\right)$. For the lower bound, use the same method as the previous item, we can also define the point $\left(\alpha_{0}, s_{1} \alpha_{0}-\right.$ $\left.\beta_{1}\left(s_{1}\right)\right)$ and $\left(\alpha_{2}, s_{2} \alpha_{2}-\beta_{2}\left(s_{2}\right)\right)$. We now replace the tangent in the previous item by the line passing through these two points. We assume the line is the graph of the linear function $y=f(x)$. Let us illustrate these relationships by figure 11 .

The proof is almost the same as before to get that for any $x \in E\left(\mu, \alpha, \alpha^{\prime}\right)$ we have $\overline{\operatorname{dim}}_{\text {loc }}\left(\mu^{\prime}, x\right) \leq f\left(\alpha^{\prime}\right)$, but we also need to use the inequality

$$
f\left(\alpha^{\prime}\right) \leq \beta_{2}^{*}\left(\alpha^{\prime}\right) \text {. }
$$

The upper bound can be obtained since $f\left(\alpha^{\prime}\right) \leq \beta_{2}^{*}\left(\alpha^{\prime}\right)$.

For the lower bound, we goes to the auxiliary measure $\mu^{\prime}$ though $\left\{p_{n}^{\prime}\right\}_{n \in \mathbb{N}}$ as the lower bound for $\operatorname{dim}_{P} \bar{E}\left(\mu, \alpha^{\prime}\right)$.

$$
p_{n}^{\prime}= \begin{cases}p^{s_{1}} A^{\beta_{1}\left(s_{1}\right)} & N_{2 i}<n \leq N_{2 i+1} \text { for some } i \in \mathbb{N} \\ q^{s_{2}} B^{\beta_{2}\left(s_{2}\right)} & N_{2 i+1}<n \leq N_{2 i+2} \text { for some } i \in \mathbb{N} .\end{cases}
$$

Let us give some remarks to the proof of theorem 2.5 .

(1) The Hausdorff dimension: The lower bound can be obtained by a suitable auxiliary measure $\mu^{\prime}$. Now we turn to the upper bound. When $\left(\alpha, \alpha^{\prime}\right) \notin\left[\beta_{1}^{\prime}(1), \beta_{1}^{\prime}(0)\right] \times$ $\left[\beta_{2}^{\prime}(+\infty), \beta_{2}^{\prime}(1)\right)$, the upper bound can be obtained by $E\left(\mu, \alpha, \alpha^{\prime}\right)=\underline{E}(\mu, \alpha) \cap$ $\bar{E}\left(\mu, \alpha^{\prime}\right)$ and theorem 2.3. But this may not give the sharp upper bound for $\alpha^{\prime} \in$ $\left[\beta_{2}^{\prime}(+\infty), \beta_{2}^{\prime}(1)\right)$, also the methods in [11, 1] fail. So we turn to use [8. Proposition 2.3] and calculate the upper bound of $\underline{\operatorname{dim}}_{\mathrm{loc}}\left(\mu^{\prime}, x\right)$ for all $x \in E\left(\mu, \alpha, \alpha^{\prime}\right)$ for a suitable measure $\mu^{\prime}$ to give the sharp upper bound.

This result is reasonable, if we compare with $\operatorname{dim}_{H}(\underline{E}(\mu, \alpha))=\min \left\{\alpha, \frac{\log 2}{\log A}\right\}$ when $\alpha \in\left[\beta_{1}^{\prime}(1), \beta_{1}^{\prime}(0)\right)$ since we use the optimal tangent $y=x$. But for the situation $E\left(\mu, \alpha, \alpha^{\prime}\right)$, it is $y=s_{1} x-\beta_{1}\left(s_{1}\right)$. By the way, when $\alpha^{\prime}$ fulfill $\left[\beta_{2}^{\prime}(+\infty), \beta_{2}^{\prime}(1)\right)$, the curve $\operatorname{dim}_{H} E\left(\mu, \alpha, \alpha^{\prime}\right)$ fulfill the gap between $\operatorname{dim}_{H}(\underline{E}(\mu, \alpha))$ and $\beta_{1}^{*}(\alpha)$ for $\alpha \in\left[\beta_{1}^{\prime}(1), \beta_{1}^{\prime}(0)\right)$. 
(2) The packing dimension: It also has a duality with the Hausdorff dimension so there is a similar result. The lower bound can be obtained by a suitable auxiliary measure $\mu^{\prime}$. Now we turn to the upper bound. When $\left(\alpha, \alpha^{\prime}\right) \notin\left[\beta_{1}^{\prime}(1), \beta_{1}^{\prime}(-\infty)\right] \times$ $\left[\beta_{2}^{\prime}(+\infty), \beta_{2}^{\prime}(1)\right)$, the upper bound can be obtained by $E\left(\mu, \alpha, \alpha^{\prime}\right)=E(\mu, \alpha) \cap$ $\bar{E}\left(\mu, \alpha^{\prime}\right)$ and theorem 2.3 . But this may not give the sharp upper bound for $\alpha \in$ $\left[\beta_{1}^{\prime}(1), \beta_{1}^{\prime}(-\infty)\right]$, also the methods in [11, 1] fail. So we turn to use [8, Proposition 2.3] and calculate the upper bound of $\overline{\operatorname{dim}}_{\text {loc }}\left(\mu^{\prime}, x\right)$ for all $x \in E\left(\mu, \alpha, \alpha^{\prime}\right)$ for a suitable measure $\mu^{\prime}$ to give the sharp upper bound.

This result is also reasonable, if we compare with $\operatorname{dim}_{P}\left(\underline{E}\left(\mu, \alpha^{\prime}\right)\right)=\alpha^{\prime}$ when $\alpha^{\prime} \in\left[\beta_{2}^{\prime}(+\infty), \beta_{2}^{\prime}(1)\right)$ since we use the optimal tangent $y=x$. But for the situation $E\left(\mu, \alpha, \alpha^{\prime}\right)$, it is $y=s_{2} x-\beta_{2}\left(s_{2}\right)$. By the way, when $\alpha$ fulfill $\left[\beta_{1}^{\prime}(1), \beta_{1}^{\prime}(-\infty)\right]$, the curve $\operatorname{dim}_{H} E\left(\mu, \alpha, \alpha^{\prime}\right)$ fulfill the gap between $\operatorname{dim}_{H}(E(\mu, \alpha))$ and $\beta_{2}^{*}(\alpha)$ for $\alpha^{\prime} \in\left[\beta_{2}^{\prime}(+\infty), \beta_{2}^{\prime}(1)\right)$.

\section{REFERENCES}

[1] J. Barral. Inverse problems in multifractal analysis of measures. Ann. Sci. Éc. Norm. Supér. (4), 48(6):14571510, 2015.

[2] J. Barral, F. Ben Nasr, and J. Peyrière. Comparing multifractal formalisms: the neighboring boxes condition. Asian J. Math., 7(2):149-165, 2003.

[3] F. Ben Nasr, I. Bhouri, and Y. Heurteaux. The validity of the multifractal formalism: results and examples. Adv. Math., 165(2):264-284, 2002.

[4] F. Ben Nasr and J. Peyrière. Revisiting the multifractal analysis of measures. Rev. Mat. Iberoam., 29(1):315-328, 2013.

[5] G. Brown, G. Michon, and J. Peyrière. On the multifractal analysis of measures. J. Statist. Phys., 66(34):775-790, 1992.

[6] R. Cawley and R. D. Mauldin. Multifractal decompositions of Moran fractals. Adv. Math., 92(2):196-236, 1992.

[7] K. L. Chung. A course in probability theory. Academic Press, Inc., San Diego, CA, third edition, 2001.

[8] K. Falconer. Techniques in fractal geometry. John Wiley \& Sons, Ltd., Chichester, 1997.

[9] D.-J. Feng and K.-S. Lau. Multifractal formalism for self-similar measures with weak separation condition. J. Math. Pures Appl. (9), 92(4):407-428, 2009.

[10] D.-J. Feng and E. Olivier. Multifractal analysis of weak Gibbs measures and phase transitionapplication to some Bernoulli convolutions. Ergodic Theory Dynam. Systems, 23(6):1751-1784, 2003.

[11] L. Olsen. A multifractal formalism. Adv. Math., 116(1):82-196, 1995.

[12] Y. Qu. Exact-dimensional property of density of states measure of sturm hamiltonian. International Mathematics Research Notices, 2018(17):5417-5454, 2018.

[13] D. A. Rand. The singularity spectrum $f(\alpha)$ for cookie-cutters. Ergodic Theory Dynam. Systems, 9(3):527$541,1989$.

[14] S. Shen. Multifractal analysis of some inhomogeneous multinomial measures with distinct analytic Olsen's $b$ and B functions. J. Stat. Phys., 159(5):1216-1235, 2015.

[15] B. Testud. Phase transitions for the multifractal analysis of self-similar measures. Nonlinearity, 19(5):1201-1217, 2006.

[16] Z. Yuan. Multifractal analysis of random weak Gibbs measures. Discrete and Continuous Dynamical Systems, 37(10):5367-5405, 2017.

E-mail address: yzhh@hust.edu.cn 